\title{
Biochar: A Synthesis of Its Agronomic Impact beyond Carbon Sequestration
}

\author{
Kurt A. Spokas, ${ }^{*}$ Keri B. Cantrell, Jeffrey M. Novak, David W. Archer, James A. Ippolito, Harold P. Collins, Akwasi \\ A. Boateng, Isabel M. Lima, Marshall C. Lamb, Andrew J. McAloon, Rodrick D. Lentz, and Kristine A. Nichols
}

Biochar has been heralded as an amendment to revitalize degraded soils, improve soil carbon sequestration, increase agronomic productivity, and enter into future carbon trading markets. However, scientific and economic technicalities may limit the ability of biochar to consistently deliver on these expectations. Past research has demonstrated that biochar is part of the black carbon continuum with variable properties due to the net result of production (e.g., feedstock and pyrolysis conditions) and postproduction factors (storage or activation). Therefore, biochar is not a single entity but rather spans a wide range of black carbon forms. Biochar is black carbon, but not all black carbon is biochar. Agronomic benefits arising from biochar additions to degraded soils have been emphasized, but negligible and negative agronomic effects have also been reported. Fifty percent of the reviewed studies reported yield increases after black carbon or biochar additions, with the remainder of the studies reporting alarming decreases to no significant differences. Hardwood biochar (black carbon) produced by traditional methods (kilns or soil pits) possessed the most consistent yield increases when added to soils. The universality of this conclusion requires further evaluation due to the highly skewed feedstock preferences within existing studies. With global population expanding while the amount of arable land remains limited, restoring soil quality to nonproductive soils could be key to meeting future global food production, food security, and energy supplies; biochar may play a role in this endeavor. Biochar economics are often marginally viable and are tightly tied to the assumed duration of agronomic benefits. Further research is needed to determine the conditions under which biochar can provide economic and agronomic benefits and to elucidate the fundamental mechanisms responsible for these benefits.

Copyright $\odot 2012$ by the American Society of Agronomy, Crop Science Society of America, and Soil Science Society of America. All rights reserved. No part of this periodical may be reproduced or transmitted in any form or by any means, electronic or mechanical, including photocopying, recording, or any information storage and retrieval system, without permission in writing from the publisher.

J. Environ. Qual. 41

doi:10.2134/jeq2011.0069

Received 1 Mar. 2011.

*Corresponding author (kurt.spokas@ars.usda.gov).

(c) ASA, CSSA, SSSA

5585 Guilford Rd., Madison, WI 53711 USA
$\mathrm{B}$ LACK CARBON (BC) is the name given to the spectrum of chemical-thermal solid conversion products formed from carbonaceous materials, which could be biomass or fossil fuels (Goldberg, 1985; Masiello et al., 2002). The BC continuum contains all charred residues, with a lack of consistency over terminology ranging from char, charcoal, bone char, carbon ash, carbon black, black carbon, carbonized carbon, coke, and soot (Jones et al., 1997; Masiello, 2004). Recently, biochar has been added to this $\mathrm{BC}$ terminology mixture. In this review, the term $B C$ is used for the carbonaceous solid byproduct of the chemical-thermal conversion of any carbon-containing material that may or may not be biomass. Biochar refers to $\mathrm{BC}$ that is produced as a vehicle of carbon sequestration from renewable and sustainable biomass (Lehmann, 2007). Therefore, biochar is $\mathrm{BC}$, but not all $\mathrm{BC}$ is biochar.

Black carbon has been applied to soils virtually from the dawn of civilization, since fire pits were built on soil, and associated research can be documented to the start of modern science (Lefroy, 1883; Hall, 1910). In addition, BC use in agriculture dates back at least to the early 1600s in Japan and potentially earlier in China (cited in Ogawa and Okimori, 2010). These purposeful BC applications, combined with the natural deposition of BC (e.g., forest fires, prairie fires, volcanoes), have resulted in the widespread presence of BC in the soil organic matter pool (Skjemstad et al., 2002). The first use of the term biochar was around 1998 for the solid residual of biomass pyrolysis (Bapat and Manahan, 1998). In the late 1980s, there was an immense shift in the intended purpose for biomass pyrolysis-from an energy and chemical resource to a means of atmospheric carbon sequestration (Goldberg, 1985; Kuhlbusch and Crutzen, 1995). This alteration of purpose has prompted a shift in referring to $\mathrm{BC}$ that is produced

K.A. Spokas, USDA-ARS, Soil and Water Management Unit; St. Paul, MN and Univ. of Minnesota, Dep. of Soil, Water and Climate, St. Paul, MN 55108; K.B. Cantrell and J.M. Novak, USDA-ARS, Coastal Plains Soil, Water, and Plant Research Center, Florence, SC; D.W. Archer and K.A. Nichols, USDA-ARS, Northern Great Plains Research Lab., Mandan, ND; J.A. Ippolito and R.D. Lentz, USDA-ARS, Northwest Irrigation and Soils Research Lab., Kimberly, ID; H.P. Collins, USDA-ARS, The Vegetable and Forage Crop Research Unit, Prosser, WA; A.A. Boateng and A.J. McAloon, USDA-ARS, Eastern Regional Research Center, Wyndmoor, PA; I.M. Lima, USDA-ARS, Southern Regional Research Center, New Orleans, LA; M.C. Lamb, USDA-ARS, National Peanut Research Lab, Dawson, GA. Trade names are necessary to report factually on available data; however, the USDA neither guarantees nor warrants the standard of the product, and the use of the name by USDA implies no approval of the product to the exclusion of others that may also be suitable. Assigned to Associate Editor Jan Willem van Groenigen.

Abbreviations: BC, black carbon; CEC, cation exchange capacity; GHG, greenhouse gas; VOC, volatile organic compound. 
for carbon sequestration purposes (assumed to be applied to soils) as biochar (Karaosmanoglu et al., 2000; Lehmann, 2007; Laird, 2008).

The current application of biochar to soil is modeled after the Amazonian Terra Preta soils, which have higher soil fertility believed to result from intentional BC additions from "slash and char" agricultural practices (Mishra and Ramakrishnan, 1983; German, 2003; Mann, 2005). However, BC additions to soils have not uniformly resulted in soil fertility improvements. Charcoal spots (historical charcoal production sites) in Zambian forests possess slower plant regeneration rates than surrounding areas without BC remnants (Chidumayo, 1988), and the long-term application of pyrolysis products to soils at historical US wood pyrolysis plants (wood distillation) that occurred from 1800 to 1960 raised soil organic chemical concentrations to such high levels that some sites are included in the U.S. Environmental Protection Agency's Superfund program (Erstfeld and Snow-Ashbrook, 1999; Edenborn and Severson, 2007). Due to these diverse potential environmental impacts arising from soil application of biomass pyrolysis products, it is important to summarize existing observations and to document current gaps in our understanding of biochar's potential impacts, both positive and negative, to guide future use.

\section{Biochar Production}

Pyrolysis refers to the process of thermochemical decomposition of organic material at elevated temperatures in the absence of oxygen (Bridgwater, 1994). There are three product streams from pyrolysis: (i) noncondensable gases, (ii) a combustible bio-oil representing the condensable liquids (tars), (iii) and biochar, a solid residual coproduct. Pyrolysis of biomass dates back at least $5000 \mathrm{yr}$ when the Egyptians formed pyroligneous acid (wood vinegar, tars, and smoke condensates or bio-oil) used for embalming (Baumann, 1960). Biomass pyrolysis products served as chemical and energy sources for the next $-5000 \mathrm{yr}$. Wood pyrolysis peaked in the early 1900s with the "standard distillation apparatus" (retort) that processed 10 cords of wood $(25 \mathrm{Mg})$ within a 24-h period (Hawley, 1926). Biomass pyrolysis was replaced by coal in the 1800 s as an energy source and by crude oil (petroleum) in the 1920s as a chemical source due to the improved economics of nonrenewable fossil fuels, the improved distillation efficiency of the higher-purity petroluem products, and the decline of woody resources in proximity to pyrolysis plants (Owen, 1975; Edenborn and Severson, 2007).

Pyrolysis can be an endothermic or exothermic reaction depending on the temperature of the reactants, becoming increasingly exothermic as the reaction temperature decreases (Mok and Antal, 1983). The exothermicity of the slow pyrolysis reaction per unit of biochar yield is reported to range from 2.0 to $3.2 \mathrm{~kJ} \mathrm{~g}^{-1}$ char (Mok and Antal, 1983; Milosavljevic et al., 1996). Hence, because the fixed carbon content of biomass is high, biochar formation commences at low temperatures where autogeneous pyrolysis begins (Mok and Antal, 1983; Milosavljevic et al., 1996). The self-sustaining nature of the low-temperature reaction explains why traditional methods of making charcoal, where biomass was buried underground, could carry on for days.
Current biochar production is focused on advanced pyrolysis systems (Bridgwater et al., 1999; Boateng et al., 2007; Zhang et al., 2007; Boateng et al., 2010b; Lima et al., 2010; Lima and Marshall, 2010). However, traditional charcoal production technologies are still used (Major et al., 2010b). Advanced conversion systems (Table 1) allow precise control of operating conditions, which, coupled with feedstock selection, can regulate the physical and chemical properties of biochar (Table 2) and allow for customization of biochar properties. Available biochar nutrients are linked to the differences in the composition of the original parent feedstock (Abdullah et al., 2010; Song and Peng, 2010; Yip et al., 2010), the moisture content of the feedstock (Yip et al., 2007), pyrolysis production conditions (Bridgwater, 1994; Bridgwater et al., 1999; Antal and Grønli, 2003; Hossain et al., 2011), and postproduction handling and processing (Azargohar and Dalai, 2008) because these factors control the degree of aromaticity and potential entrapment of noncarbon atoms in $\mathrm{BC}$ carbon ring structures. The resulting biochar nutrient contents are variable based on feedstock and production conditions, but this has been known for some time (Anonymous, 1840) (Table 2). Furthermore, differences have been noted in the chemistries of various biochars, despite the similarity in production conditions (DeGroot et al., 1991; Bustin and Guo, 1999). Recent advancements in pyrolysis conversions, particularly the process controls, have reduced this variability (Cantrell and Martin, 2011).

Further discussion of the various production techniques and impacts on the product yields are found in the associated references in Table 1. These are average ranges of the physical properties and yields, but quantities vary widely as a function of feedstock and presence of impurities (e.g., soil) (Table 2) (Spokas et al., 2011). Slow pyrolysis is the conversion technique that maximizes biochar yield, but the other variants of hydrothermal carbonization and microwave-assisted pyrolysis are also appealing due to their ability to handle wetter biomass sources, which reduces biomass drying costs (Table 1). Torrefaction had been established in the forestry industry for fuel upgrading of woody biomass, primarily for densification, reducing transportation costs due to moisture removal and increasing heating values (Bourgois and Guyonnet, 1988), which generates an energy product amenable to coal-blending and subsequent co-combustion (Bridgeman et al., 2010; Repellin et al., 2010; Phanphanich and Mani, 2011). Torrefaction is a mild form of pyrolysis, with production temperatures ranging between 200 and $300^{\circ} \mathrm{C}$ (Prins et al., 2006). However, torrefaction and hydrothermal carbonization are not optimal choices for biochar production because the oxygen to carbon $(\mathrm{O} / \mathrm{C})$ ratio of the resulting charred material is high (between 0.4 and 0.6) (Bridgeman et al., 2008), indicating lower aromatic character in the biochar (Fuertes et al., 2010). When biochar is produced at higher pyrolysis temperatures $\left(400-700^{\circ} \mathrm{C}\right)$, its carbon is distributed in more polycondensed aromatic structures (Keiluweit et al., 2010). These polycondensed aromatic structures have low $\mathrm{O} / \mathrm{C}$ ratios that are resistant to microbial degradation (Glaser et al., 2002; Kimetu and Lehmann, 2010; Zimmerman, 2010). These properties are important because biochar that resists microbial mineralization may be best suited for long-term soil carbon sequestration. 
Characteristics of the raw feedstock biomass impart specific properties to the resulting biochar, such as ash content and its elemental constituents, density, and hardness. Biomass with high elemental contents usually produces an even higher elemental concentration in the ash portion of biochar (Abdullah et al., 2010). Particularly, biochars from grass, corn stover, and manure feedstocks contain higher amounts of ash than biochars from other biomass sources (Brewer et al., 2009) (Table 2). However, the ash content also can be reduced by postproduction activities, such as acid washing (DeGroot et al., 1991). Specific biochar nutrient concentrations may be greater (Abdullah et al., 2010) or lower (DeGroot et al., 1991) than original feedstock nutrient concentrations. These results suggest that occasional volatilization and loss of nutrients during pyrolysis may be linked to higher production temperatures (Cantrell and Martin, 2011). The large range of operational maximum temperatures common to slow pyrolysis processes determines the extent of volatilization taking place and therefore the final composition of the resulting biochar in terms of residual volatile content. During pyrolysis, a series of cleavage and polymerization reactions occurs, resulting in the formation of fixed carbon (aromatic) structures that are thermally stable. Devolatilization and carbonization processes are involved, as described elsewhere (Aiman and Stubington, 1993; Sjöström, 1993; Alén et al., 1996; Drummond and Drummond, 1996). Biochars created from grasses and manures (including poultry litter) appear to possess higher nutrient contents than other feedstocks (Table 2).

Historically, BC has been an undesirable waste product because the primary focus has been on optimizing the liquid and gas products for energy conversion and not on biochar for carbon sequestration. Despite the long research history of pyrolysis, further research is needed into the techniques to optimize biochar yields. Biochar is not homogenous or a single material; differing nutrient and chemical structures and differing positions in the $\mathrm{BC}$ spectrum lead to the lack of a "one-size-fits-all" biochar (Novak and Busscher, 2011). A holistic and objective approach to the production of biochar is vital for economic success where profits from all co-products are optimized. Thus, for a biochar to deliver an agronomic benefit, it is important to understand how biochar quality (physical and chemical properties) is influenced by the choice of feedstock and the pyrolysis conditions used in its production (Antal and Grønli, 2003; Lehmann and Joseph, 2009; Novak et al., 2009b).

\section{Biochar Impacts on Agronomic Yields}

Soil fertility is influenced by a number of soil properties and involves a complex balance of biotic and abiotic reactions that are spatially and temporally dynamic. Adding biochar to soils may produce immediate effects on properties such as soil nutrition, water retention, or microbial activity (Atkinson et al., 2010; Lehmann et al., 2011), although these effects vary

Table 1. Description of advanced thermal conversion processes. $\dagger$

\begin{tabular}{|c|c|c|c|c|c|c|c|c|c|c|c|}
\hline \multirow{2}{*}{$\begin{array}{l}\text { Conversion } \\
\text { type }\end{array}$} & \multirow{2}{*}{$\begin{array}{l}\text { Temp. } \\
\text { range }\end{array}$} & \multirow{2}{*}{$\begin{array}{l}\text { Residency } \\
\text { time }\end{array}$} & \multirow{2}{*}{$\begin{array}{l}\text { Heating } \\
\text { rate }\end{array}$} & \multicolumn{3}{|c|}{$\begin{array}{l}\text { Product production (\% of } \\
\text { original feedstock mass) }\end{array}$} & \multicolumn{4}{|c|}{ Solid proximate analysis } & \multirow[t]{2}{*}{ Other notes } \\
\hline & & & & Solid & Liquid & Gas & Moisture & VM & Ash & Fixed C & \\
\hline & ${ }^{\circ} \mathrm{C}$ & & ${ }^{\circ} \mathrm{C} \mathrm{s}^{-1}$ & & & & $\%-$ & & & - & \\
\hline Torrefaction & $200-320$ & hours & $<1$ & $40-90$ & - & $10-60$ & $0-1$ & $50-85$ & $2-10$ & $13-38$ & $\begin{array}{l}\text { densification and increase energy } \\
\text { value of solid residuals; energy } \\
\text { generation }\end{array}$ \\
\hline Slow pyrolysis & $350-700$ & hours & $1-100$ & $15-40$ & $20-55$ & $20-60$ & $0-5$ & $5-20$ & $2-10$ & $40-90$ & $\begin{array}{l}\text { devolatilization and } \\
\text { polymerization reactions occur; } \\
\text { maximum solid (biochar) yield }\end{array}$ \\
\hline Fast pyrolysis & $450-550$ & $<1 \mathrm{~min}$ & $>1000$ & $10-30$ & $50-70$ & $5-15$ & $0-5$ & 40 & 30 & $40-60$ & $\begin{array}{l}\text { typically fine powders (300-400 } \\
\mu \mathrm{m}) \text {; dust problems maximize } \\
\text { bio-oil production }\end{array}$ \\
\hline Flash pyrolysis & $300-800$ & $<1 \mathrm{~s}$ & $\begin{array}{l}\text { Similar to } \\
\text { fast }(>1000)\end{array}$ & $30-40$ & - & $60-70$ & $0-5$ & $5-26$ & $0-40$ & $40-60$ & $\begin{array}{l}\text { high VM potential negatives for } \\
\text { soil/plant } \\
\text { gas/solid production; no liquid } \\
\text { (oil); } \\
\text { elevated ( 1 MPa) }\end{array}$ \\
\hline Gasification & $>800$ & $\begin{array}{l}\text { seconds to } \\
\text { minutes }\end{array}$ & Variable & $0-10$ & - & $90-100$ & $\mathrm{n} / \mathrm{a}$ & $\mathrm{n} / \mathrm{a}$ & $\mathrm{n} / \mathrm{a}$ & $\mathrm{n} / \mathrm{a}$ & $\begin{array}{l}\text { ashes: } \mathrm{pH} \text { and potential toxicity } \\
\text { issues; conversion of biomass } \\
\text { to energy (no biochar or liquid } \\
\text { products) }\end{array}$ \\
\hline $\begin{array}{l}\text { Hydrothermal } \\
\text { carbonization }\end{array}$ & $150-400$ & $\begin{array}{l}\text { Minutes to } \\
\text { hours }\end{array}$ & $\mathrm{n} / \mathrm{a}$ & $5-40$ & $20-40$ & $2-10$ & $10-40$ & $50-90$ & $5-15$ & $4-10$ & $\begin{array}{l}\text { chars less stable (higher O:C } \\
\text { ratios); very high pressures (>5 } \\
\mathrm{MPa)} \\
\text { handles wet biomass }\end{array}$ \\
\hline $\begin{array}{l}\text { Microwave- } \\
\text { assisted } \\
\text { pyrolysis }\end{array}$ & $300-500$ & $\begin{array}{l}\text { minutes to } \\
\text { hours }\end{array}$ & $\mathrm{n} / \mathrm{a}$ & $20-30$ & $0-20$ & $50-70$ & $10-25$ & $20-30$ & $20-25$ & $50-60$ & $\begin{array}{l}\text { higher gas yields from microwave } \\
\text { assisted pyrolysis; handles wet } \\
\text { biomass }\end{array}$ \\
\hline
\end{tabular}

† References: Torrefacation (Bridgeman et al., 2008; Repellin et al., 2010; Phanphanich and Mani, 2011); slow pyrolysis (Apaydin-Varol et al., 2007; Pütün et al., 2007; Boateng et al., 2010b; Lima and Marshall, 2010); fast pyrolysis (Boateng, 2007; Boateng et al., 2010a; Boateng et al., 2010b; Lima et al., 2010; Mullen et al., 2010); flash (Antal and Grønli, 2003; Deenik et al., 2010); gasification (Masclet et al., 1987; Ptasinski, 2008; Salleh et al., 2010; FernándezPereira et al., 2011); hydrothermal carbonization (Molton et al., 1981; Karagöz et al., 2005; Steinbeiss et al., 2009; Yuan et al., 2009; Rillig et al., 2010); microwave-assisted pyrolysis (Menéndez et al., 2006; Huang et al., 2008; Lei et al., 2009).

‡ Volatile matter (VM), ash content, and fixed carbon expressed on a dry weight basis. 
depending on soil type (Tryon, 1948; Shneour, 1966; Spokas and Reicosky, 2009; Van Zwieten et al., 2010b). Nonetheless, because of its generally recalcitrant nature, biochar may also have long-term impacts on soil environments. Soil formation is the net result of several external and internal factors that influence or drive pedogenic processes (Jenny, 1946; Buol et al., 2003). Biochar potentially can influence soil-forming processes that govern the accumulation, transformation, and translocation of soil constituents and hence in the long term can modify soil pedogenic activity, morphology, and productivity (Richter, 2007). For biochar to serve a beneficial role in revitalizing nutrient-impoverished soils, there should be a noted increase in the quantity of plant-available nutrients and its nutrition retention capacity (McLauchlan, 2006; Sohi et al., 2010). To understand soil-biochar interactions, we must consider how these effects vary geographically and temporally.
Assemblages of soil microbial communities and their interaction with organic and inorganic plant nutrient turnover processes are complex (Ingham et al., 1985; Zak et al., 2003) and have a profound impact on soil functions and its fertility. Microbial diversity is altered in response to organic amendments (Pérez-Piqueres et al., 2006; Sullivan et al., 2006; Khodadad et al., 2011). Research has suggested that soil applications of biochar can have a significant impact on microbial C metabolism and population dynamics (Warnock et al., 2007; O'Neill et al., 2009; Ball et al., 2010; Warnock et al., 2010; Zimmerman et al., 2011). A number of explanations for these impacts have been offered, such as biochar sorption, including the presence of volatile organic compounds (VOCs) that can inhibit or stimulate microbial mineralization reactions or affect plant-microbial interactions (Graber et al., 2010; Spokas et al., 2010), variability in biochar's susceptibility to mineralization

Table 2. Biochars chemical and fertilizer equivalent ratios. $\dagger$

\begin{tabular}{|c|c|c|c|c|c|c|c|}
\hline \multirow{2}{*}{ Reference } & \multirow{2}{*}{ Feedstock } & \multirow{2}{*}{ Pyrolysis } & \multirow{2}{*}{$\begin{array}{c}\% \text { Ash } \\
\text { (dry weight basis) }\end{array}$} & \multirow{2}{*}{$\mathrm{pH}$} & \multicolumn{3}{|c|}{ Fertilizer equivalent ratio } \\
\hline & & & & & $\mathbf{N}$ & $\mathbf{P}$ & $\mathrm{K}$ \\
\hline & \multicolumn{3}{|c|}{${ }^{\circ} \mathrm{C}$} & & \multicolumn{3}{|c|}{ kg per tonne of biochar } \\
\hline \multirow[t]{10}{*}{ Novak et al., 2009b } & peanut hull & 400 & 8.2 & 7.9 & 30 & 3 & 20 \\
\hline & peanut hull & 500 & 9.3 & 8.6 & 30 & 3 & 20 \\
\hline & pecan shell & 350 & 2.4 & 5.9 & 3 & 0.3 & 2 \\
\hline & pecan shell & 700 & 5.2 & 7.2 & 5 & 0.5 & 5 \\
\hline & poultry litter & 350 & 35.9 & 8.7 & 50 & 30 & 60 \\
\hline & poultry litter & 700 & 52.4 & 10.3 & 30 & 40 & 90 \\
\hline & switchgrass & 250 & 2.6 & 5.4 & 4 & 1 & 5 \\
\hline & switchgrass & 500 & 7.8 & 8.0 & 10 & 2 & 10 \\
\hline & hardwoods & $450-600$ & 8.9 & 5.7 & 3 & 0.3 & 6 \\
\hline & pine chips & 465 & 5.6 & 6.1 & 3 & 0.8 & 4 \\
\hline \multirow[t]{2}{*}{ Brewer et al., 2009} & corn stover & 500 & 49.7 & n/ał & 16 & 3 & 12 \\
\hline & hardwood & 500 & 13.9 & $\mathrm{n} / \mathrm{a}$ & 12 & 0.2 & 2 \\
\hline \multirow[t]{10}{*}{ Singh et al., 2010a } & wood (Eucalyptus saligna) & 400 (activated) & 4.2 & 7.7 & 2 & 0.1 & 2 \\
\hline & wood (E. saligna) & 550 (activated) & 4.4 & 9.5 & 2 & 0.2 & 2 \\
\hline & wood (E. saligna) & 400 & 3.2 & 6.9 & 2 & 0.1 & 1 \\
\hline & wood (E. saligna) & 550 & 4.4 & 8.8 & 3 & 0.2 & 2 \\
\hline & leaves (E. saligna) & 400 (activated) & 10 & 9.2 & 16 & 2 & 13 \\
\hline & leaves (E. saligna) & 550 (activated) & 11.8 & 9.8 & 17 & 3 & 15 \\
\hline & paper sludge & 550 (activated) & 65.4 & 9.2 & 2 & 0.4 & 0.5 \\
\hline & poultry manure & 400 & 42.3 & 9.2 & 52 & 6 & 25 \\
\hline & cow manure & 400 & 70.3 & 9.0 & 14 & 4 & 26 \\
\hline & cow manure & 550 & 76.2 & 8.9 & 11 & 5 & 23 \\
\hline \multirow[t]{8}{*}{ Cantrell et al., unpublished } & dairy manure & 350 & 24.2 & 9.2 & 30 & 10 & 14 \\
\hline & dairy manure & 700 & 39.5 & 9.9 & 17 & 17 & 23 \\
\hline & feedlot manure & 350 & 28.7 & 9.1 & 34 & 11 & 32 \\
\hline & feedlot manure & 700 & 44.0 & 10.3 & 17 & 18 & 49 \\
\hline & poultry litter & 350 & 30.7 & 8.7 & 53 & 21 & 49 \\
\hline & poultry litter & 700 & 46.2 & 10.3 & 22 & 31 & 74 \\
\hline & turkey litter & 350 & 34.8 & 8.0 & 43 & 26 & 40 \\
\hline & turkey litter & 700 & 49.9 & 9.9 & 20 & 37 & 56 \\
\hline \multirow[t]{2}{*}{ Cantrell and Martin, 2011} & swine manure & 350 & 32.5 & 8.2 & 37 & 39 & 18 \\
\hline & swine manure & 700 & 52.9 & 8.2 & 26 & 59 & 26 \\
\hline \multirow[t]{2}{*}{ van Zwieten et al., 2010a } & sludge + wood chip (49\%) & 550 & $\mathrm{n} / \mathrm{a}$ & 9.4 & 5 & $\mathrm{n} / \mathrm{a}$ & 0.4 \\
\hline & sludge + wood chip (69\%) & 550 & $\mathrm{n} / \mathrm{a}$ & 8.2 & 3 & $\mathrm{n} / \mathrm{a}$ & 19 \\
\hline
\end{tabular}

†These fertilizer equivalent ratios were based on the total element concentration and likely do not reflect true effective plant availability following soil application.

₹ Data not available in the reference. 
(Novak et al., 2009b; Zimmerman, 2010), microbial habitat through pH modifications (Atkinson et al., 2010), beneficial micropores on the charcoal for microbial habitat (Warnock et al., 2007), or the presence of critical nutrients for microbial growth and metabolic energy transfer reactions (GarciaMontiel et al., 2000). These and other microbial impacts have been reviewed elsewhere (Atkinson et al., 2010; Lehmann et al., 2011). The previous list highlights the importance of understanding the interactions of biochar with soil microbes, and this knowledge is vital to improve soil quality while raising crop productivity.

The past literature indicates an early interest in the use of BC to improve soil and crop growth (Lefroy, 1883). Past studies report that BC's effect on agronomic crop yield is variable, with production improvements ranging from negative to more than twofold over nonamended controls (Table $3)$. In 1833 , there was a recommendation to slowly smother burning biomass under a soil cover and then to rapidly collect the $\mathrm{BC}$ and immediately apply it to improve agronomic performance (application rate $-0.54 \mathrm{~kg}$ charcoal $\mathrm{m}^{-2}$ ) (referenced in Lefroy, 1883).

More recent biochar studies have yielded contrary results in soil quality and yield improvements (Table 3). A meta-analysis by Verheijen et al. (2009) predicted a short-term yield improvement of $12 \%$ from biochar additions, although this analysis included a limited subset of nine recent biochar specific studies (since 2007). However, there was limited accountability for the different biochar types across the different studies because biochar itself possesses a wide range of chemistries (Table 1). Biochar and $\mathrm{BC}$ additions have not consistently resulted in increased yields (Gundale and DeLuca, 2007; Rajkovich, 2010; Van Zwieten et al., 2010b). Without knowledge of the fundamental driving factors resulting in these decreased yields, our ability to extract statistically significant conclusions from existing studies is limited. From laboratory incubations, grass and nonwoody biomass biochar is more easily mineralized than wood-derived biochar, resulting in longer predicted soil residency times for wood biochar (Zimmerman, 2010). From a soil fertility perspective, this increased mineralization could provide nutrient resources to plants. On the other hand, food waste biochar (Rajkovich, 2010) and biochar with high volatile matter contents (Deenik et al., 2010) have also suppressed plant growth.

Approximately $50 \%$ of the compiled studies observed short-term positive yield or growth impacts, 30\% reported no significant differences, and $20 \%$ noted negative yield or growth impacts (Table 3). However, due to potential publication biases, these percentages should only be taken as reflective of the studies presented here and not as evidence of an overall biochar likelihood of producing positive impacts (Møller and Jennions, 2001). There are a greater number of increased yield results reported for biochar additions that occurred in weathered or degraded soils having limited fertility and productivity (Table 3). Of the $50 \%$ of the compiled studies with positive yield improvements, a majority of the yield improvements have been realized from (i) traditional kiln-formed hardwood charcoal or (ii) chars that possess plant nutrients (e.g., high $\mathrm{N}$ content in poultry manure biochar). This observation was also recently stated by Haefele et al. (2011), who observed yield increases in rice of 16 to $35 \%$ with rice hull biochar in a nutrient-poor soil compared with larger increased rice yields reported using wood biochar in similar soils (Table 3).

Numerous potential reasons exist for this apparent improved performance of traditional hardwood charcoal biochar. First is the low availability of advanced pyrolysis units. This limited availability results in a bias in the literature, with a majority of the studies using traditional charcoal techniques for the creation of biochar (Table 3).

Second, biochars from fast pyrolysis units have been extremely variable. Recently, it has been suggested that this variability could result from the incomplete conversion of the biomass feedstock due to thermal limitations and irreproducibility of heat transfer (Bruun et al., 2011). Deenik et al. (2010) also noted variable volatile matter content in fast pyrolysis biochars. This translates to differences between batches of biochar, making them potentially unique despite similar production conditions.

Last, there are differences not only in biochar quality as a function of the production process but also linked to the postproduction storage or activation (Azargohar and Dalai, 2008; Nuithitikul et al., 2010). Activation can occur by simply cooling the biochar with water or exposing the hot biochar to atmospheric oxygen during cooling. Surface oxidation of $\mathrm{BC}$, even at ambient conditions, alters surface chemical groups (Puri et al., 1958; Allardice, 1966; Cheng et al., 2006), which correspondingly influences the potential interactions with soil nutrient cycles (Bohn et al., 1985). Traditional soil kiln charcoal can be oxidized due to the exposure of the hot biochar to atmospheric air. However, often the postproduction handling of the biochar is not documented, which highlights the need for improved reporting of biochar postproduction handling and storage conditions.

\section{Potential Responsible Mechanisms for Biochar Yield Responses}

Recent studies have indicated a complex biochar and fertilizer interaction with respect to yield response (Chan et al., 2007). However, alterations in soil nutrient concentrations have not been able to fully predict yield increases (Turner, 1955; Gundale and DeLuca, 2007; Kimetu et al., 2008; Graber et al., 2010), suggesting involvement of other soil processes or properties. Biochar additions to infertile soils have been cited to improve soil cation exchange capacity (CEC) properties (Cheng et al., 2006; Liang et al., 2006; Grossman et al., 2010; Inyang et al., 2010; Lee et al., 2010). However, not all biochar-soil combinations cause an increase in CEC because no or minimal changes in CEC have also been observed after certain biochar additions to soils (Novak et al., 2009a; Nguyen et al., 2010) that have been linked to biochar production parameters (Singh et al., 2010a). Other studies have found that biochar addition may alter $\mathrm{pH}$ levels and the availability of soil nutrients such as $\mathrm{Ca}$ or $\mathrm{Mg}$, which were found to limit maize growth in highly weathered tropical soils (Major et al., 2010a), or the availability of $\mathrm{B}$ and Mo, which are important cofactors in biological $\mathrm{N}$ fixation (Rondon et al., 2007), while decreasing exchangeable $\mathrm{Al}^{3+}$ and $\mathrm{H}^{+}$concentrations (Novak et al., 2009a). 
Table 3. Impacts of black carbon and biochar additions on the yield of various crops.

\begin{tabular}{|c|c|c|c|c|c|}
\hline Reference & Country & Soil type & Crop & Addition/rate & $\begin{array}{c}\text { Yield results } \\
\text { (compared with control) }\end{array}$ \\
\hline \multirow[t]{2}{*}{ Asai et al., 2009} & Laos & Laotian paddy soils; field plots & rice & charcoal (various) & $\begin{array}{l}\text { higher grain yields at sites } \\
\text { with low P availability } \\
\text { with biochar }\end{array}$ \\
\hline & Laos & Laotian paddy soils; field plots & rice & charcoal (various) & $\begin{array}{l}\text { reduced grain yields } \\
\text { in soils with a low } \\
\text { indigenous N supply }\end{array}$ \\
\hline \multirow[t]{3}{*}{$\begin{array}{l}\text { Bovey and Miller, } \\
1969\end{array}$} & U.S. & Toa silty clay + sand & $\begin{array}{c}\text { beans (Phaseolus } \\
\text { vulgaris L.) }\end{array}$ & $\begin{array}{l}\text { activated charcoal } \\
\quad\left(640 \mathrm{mg} \mathrm{kg}^{-1}\right)\end{array}$ & $+26 \%$ (yield increase) \\
\hline & U.S. & sand & cucumbers & $\begin{array}{l}\text { activated charcoal } \\
\quad\left(640 \mathrm{mg} \mathrm{kg}^{-1}\right)\end{array}$ & $-15 \%$ (yield reduction) \\
\hline & U.S. & Toa silty clay & cucumbers and oats & $\begin{array}{l}\text { activated charcoal } \\
\quad(0-1 \% \mathrm{w} / \mathrm{w})\end{array}$ & $\begin{array}{l}+54 \% \text { oats }+77 \% \\
\text { cucumbers }\end{array}$ \\
\hline Chen et al., 2010 & Japan & Shimajiri maji soil (heavy clay) & sugarcane & $\begin{array}{l}\text { sugarcane bagasse } \\
\text { biochar }(3 \%+ \\
\text { fertilizer) }\end{array}$ & increased sugarcane yield \\
\hline $\begin{array}{l}\text { Colauto et al., } \\
2010\end{array}$ & Brazil & compost/soil & $\begin{array}{c}\text { mushroom (Agaricus } \\
\text { brasiliensis) }\end{array}$ & $\begin{array}{l}\text { charcoal (?) (charcoal } \\
\text { as casing layer) }\end{array}$ & $-50 \%$ \\
\hline $\begin{array}{l}\text { Constantin et al., } \\
1977\end{array}$ & U.S. & culture media & $\begin{array}{c}\text { tobacco (Nicotiana } \\
\text { tabacum) }\end{array}$ & activated charcoal & $\begin{array}{l}\text { sorbs plant hormones, } \\
\text { inhibiting callus and shoot } \\
\text { development (negative } \\
\text { effects observed) }\end{array}$ \\
\hline \multirow[t]{2}{*}{$\begin{array}{l}\text { de Keijzer and } \\
\text { Hermann, } 1966\end{array}$} & U.S. & laboratory/field various & conifer species & charcoal (various) & $\begin{array}{l}\text { summarizes impact on } \\
\text { germination of conifer } \\
\text { species (positive, } \\
\text { negative, and no impact) }\end{array}$ \\
\hline & & field plot & douglas-fir & charcoal (various) & $\begin{array}{l}\text { increased germination } \\
\text { tied to increased soil } \\
\text { temperature }\end{array}$ \\
\hline $\begin{array}{l}\text { Deenik et al., } \\
2010\end{array}$ & U.S. & greenhouse/lab & lettuce and corn & $\begin{array}{l}\text { fast pyrolysis } \\
\text { macadamia nut } \\
\text { shell }(0-20 \% \text { by wt) }\end{array}$ & yield decreases observed \\
\hline Devonald, 1982 & U.K. & growing media & $\begin{array}{c}\text { garden peas (Pisum } \\
\text { sativum) }\end{array}$ & $\begin{array}{l}\text { activated charcoal } \\
(5 \% \mathrm{w} / \mathrm{w})\end{array}$ & $\begin{array}{l}\text { significant decrease in } \\
\text { shoot height/rot mass } \\
\text { and nodulation in peas }\end{array}$ \\
\hline \multirow[t]{2}{*}{$\begin{array}{l}\text { Gaskin et al., } \\
2010\end{array}$} & U.S. & $\begin{array}{l}\text { Tifton loamy sand soil (Plinthic } \\
\text { Kandiudult) }\end{array}$ & corn & $\begin{array}{c}\text { pine chip biochar }(0, \\
\left.11.2,22.4 \mathrm{Mg} \mathrm{ha}^{-1}\right)\end{array}$ & $\begin{array}{l}\text { 2006: decrease with } \\
\text { increasing } B C+; \\
\text { 2007: increase with BC } \\
\text { amounts }\end{array}$ \\
\hline & U.S. & $\begin{array}{l}\text { Tifton loamy sand soil (Plinthic } \\
\text { Kandiudult) }\end{array}$ & corn & $\begin{array}{l}\text { peanut hull biochar } \\
(0,11.2,22.4 \mathrm{Mg} \\
\left.\mathrm{ha}^{-1}\right)\end{array}$ & $\begin{array}{l}\text { decreases/increases; } \\
\text { no statistically significant } \\
\text { pattern }\end{array}$ \\
\hline \multirow[t]{2}{*}{$\begin{array}{l}\text { Gundale and } \\
\text { DeLuca, } 2007\end{array}$} & U.S. & $\begin{array}{c}\text { sandy-skeletal, mixed, frigid Typic } \\
\text { Dystrustepts }\end{array}$ & $\begin{array}{c}\text { perennial grass (Koeleria } \\
\text { macrantha) }\end{array}$ & $\begin{array}{l}\text { laboratory produced } \\
\text { charcoal }\left(350^{\circ} \mathrm{C}, 2\right. \\
\text { h); various rates }\end{array}$ & $\begin{array}{l}\text { yield suppressions } \\
\text { (Conclusion: Differences } \\
\text { existed between wildfire } \\
\text { charcoal and laboratory } \\
\text { created charcoal.) }\end{array}$ \\
\hline & & & & $\begin{array}{l}\text { wildfire charcoal, } \\
\text { various }\end{array}$ & $\begin{array}{l}\text { yield increases (correlated } \\
\text { with amount of charcoal) }\end{array}$ \\
\hline \multirow[t]{3}{*}{$\begin{array}{l}\text { Haefele et al., } \\
2011\end{array}$} & Philippines & anthraquic Gleysols & rice & $\begin{array}{l}\text { rice husk biochar } \\
\text { (traditional) }\end{array}$ & $\begin{array}{l}\text { initial negative; after fourth } \\
\text { season no significant effect }\end{array}$ \\
\hline & Philippines & Humic Nitisols & rice & rice husk (traditional) & no significant effect \\
\hline & Thailand & Gleyic acrisols & rice & $\begin{array}{l}\text { rice husk biochar } \\
\text { (traditional) }\end{array}$ & $\begin{array}{l}\text { positive effects; poorest } \\
\text { soil and most draft stress } \\
16-35 \% \text { yield increase }\end{array}$ \\
\hline Herr et al., 1999 & U.S. & forest soil & white pine (Pinus strobus L.) & lab burnt wood ash & no differences \\
\hline $\begin{array}{l}\text { Hossain et al., } \\
2010\end{array}$ & Australia & chromosol & $\begin{array}{c}\text { tomato (Lycopersicon } \\
\text { esculentum) }\end{array}$ & $\begin{array}{l}\text { wastewater sludge } \\
\text { biochar }\left(10 \mathrm{t} \mathrm{ha}^{-1}\right)\end{array}$ & $\begin{array}{l}+64 \% \text { with fertilizer } \\
\text { additions }\end{array}$ \\
\hline \multirow[t]{6}{*}{$\begin{array}{l}\text { Iswaran et al., } \\
1980\end{array}$} & India & $\begin{array}{c}\text { Delphi agricultural soil (no } \\
\text { description) }\end{array}$ & moong (Vigna radiata) & $\begin{array}{l}\text { charcoal } \\
\left(500 \mathrm{~kg} \mathrm{ha}^{-1}\right)\end{array}$ & $+20 \%$ \\
\hline & & soybean (Glycine max) & charcoal (500 kg ha $\left.{ }^{-1}\right)$ & $+50 \%$ & \\
\hline & & pea (Pisum sativum) & charcoal (500 kg ha $\left.{ }^{-1}\right)$ & $+60 \%$ & \\
\hline & & moong (V. radiata) & coal (500 kg ha-1) & $+30 \%$ & \\
\hline & & soybean (G. max) & coal (500 kg ha $\left.{ }^{-1}\right)$ & $+140 \%$ & \\
\hline & & pea (P. sativum) & coal (500 kg ha-1) & $+70 \%$ & \\
\hline
\end{tabular}


Table 3. Continued.

\begin{tabular}{|c|c|c|c|c|c|}
\hline Reference & Country & Soil type & Crop & Addition/rate & $\begin{array}{c}\text { Yield results } \\
\text { (compared with control) }\end{array}$ \\
\hline $\begin{array}{l}\text { Kadota and Niimi, } \\
2004\end{array}$ & Japan & potting mix & bedding plants & charcoal + PA & $\begin{array}{l}\text { negative growth shown } \\
\text { in French marigold and } \\
\text { scarlet sage; positive } \\
\text { effects for melampodium, } \\
\text { scarlet sage, and zinnia }\end{array}$ \\
\hline Kim et al., 2003 & Korea & unknown & red pepper & charcoal & $\begin{array}{l}\text { small particle size: } \\
\text { increased yield; large } \\
\text { particle charcoal: } \\
\text { decreased root growth; } \\
\text { no yield differences }\end{array}$ \\
\hline $\begin{array}{l}\text { Kimetu et al., } \\
2008\end{array}$ & Kenya & Ultisol & corn & $\begin{array}{l}\text { biochar (traditional } \\
\text { kiln) Eucalyptus } \\
\text { saligna ( } 7 \text { tons BC } \\
\text { ha }^{-1} \text { ) }\end{array}$ & +80 to $+100 \%$ \\
\hline $\begin{array}{l}\text { Kratky and Warren, } \\
1971\end{array}$ & U.S. & $\begin{array}{l}\text { vermiculite }+ \text { activated carbon } \\
\text { (greenhouse) }\end{array}$ & $\begin{array}{l}\text { cucumbers (Cucumis } \\
\text { sativus L.) }\end{array}$ & $\begin{array}{l}\text { activated charcoal } \\
(7 \% \mathrm{w} / \mathrm{w})\end{array}$ & no differences \\
\hline $\begin{array}{l}\text { Kratky and Warren, } \\
1971\end{array}$ & U.S. & vermiculite + activated carbon & $\begin{array}{l}\text { tomatoes (Lycopersicum } \\
\text { esculentum) }\end{array}$ & $\begin{array}{l}\text { activated charcoal } \\
7 \%(\mathrm{w} / \mathrm{w})\end{array}$ & no differences \\
\hline $\begin{array}{l}\text { Kratky and Warren, } \\
1971\end{array}$ & U.S. & field plot soil (Indiana soil) & tomatoes (L. esculentum) & $\begin{array}{l}\text { activated charcoal } \\
7 \%(w / w)\end{array}$ & no differences \\
\hline $\begin{array}{l}\text { Kulmatiski and } \\
\text { Beard, } 2006\end{array}$ & U.S. & $\begin{array}{l}\text { coarse-loamy, mixed mesic typic } \\
\text { haploxerolls (field plots) }\end{array}$ & $\begin{array}{l}\text { native and exotic grassland } \\
\text { vegetation }\end{array}$ & $\begin{array}{l}\text { activated charcoal } \\
(1 \% \mathrm{w} / \mathrm{w})\end{array}$ & $\begin{array}{l}\text { no differences first year; } \\
\text { second year: increased } \\
\text { plant cover, heterotrophic } \\
\text { bacteria differences noted }\end{array}$ \\
\hline $\begin{array}{l}\text { Lamb et al., } \\
\text { unpublished }\end{array}$ & U.S. & $\begin{array}{l}\text { Greenville fine sandy loam (fine, } \\
\text { kaolinitic, thermic Rhodic } \\
\text { Kandiudults) }\end{array}$ & peanut, corn, and cotton & $\begin{array}{l}\text { fast pyrolysis, } \\
\text { hardwood }(22,500 \\
\left.\& 45,000 \mathrm{~kg} \mathrm{ha}^{-1}\right)\end{array}$ & $\begin{array}{l}\text { no difference (year 1- } \\
\text { ongoing) }\end{array}$ \\
\hline Lau et al., 2008 & various & various & various & $\begin{array}{l}\text { various activated } \\
\text { charcoals, various } \\
\text { rates }\end{array}$ & $\begin{array}{l}\text { positive and negative; } \\
\text { mostly positive effects } \\
\text { observed }\end{array}$ \\
\hline Leibundgut, 1960 & Germany & litter compost & conifer & $\begin{array}{l}\text { charcoal (beech } \\
\text { wood) }(0.5-2 \mathrm{~kg} \\
\left.\mathrm{m}^{-2}\right)\end{array}$ & $\begin{array}{l}\text { no significant effect on } \\
\text { germination; some } \\
\text { inhibition on conifer } \\
\text { seedling growth observed }\end{array}$ \\
\hline $\begin{array}{l}\text { Linscott and } \\
\text { Hagin, } 1967\end{array}$ & U.S. (NY) & Lima silty clay loam & alfalfa & $\begin{array}{l}\text { activated charcoal ( } 8 \\
\text { lbs per acre) }\end{array}$ & $\begin{array}{l}50 \% \text { increase; not } \\
\text { statistically significant }\end{array}$ \\
\hline $\begin{array}{l}\text { Mohamed- } \\
\text { Yasseen, } 2001\end{array}$ & Egypt & culture media & corn & $\begin{array}{l}\text { activated charcoal (5 } \\
\left.\mathrm{g} \mathrm{L}^{-1}\right)\end{array}$ & $\begin{array}{l}\text { longer shoots and roots in } \\
\text { the presence of charcoal }\end{array}$ \\
\hline $\begin{array}{l}\text { Namgay et al., } \\
2010\end{array}$ & Australia & Quartzipsamment & corn & $\begin{array}{l}\text { wood; slow pyrolysis } \\
\left(550^{\circ} \mathrm{C}\right)(0,10 \text {, and } \\
\left.50 \mathrm{mg} \mathrm{kg}^{-1}\right)\end{array}$ & $\begin{array}{l}\text { no significant differences } \\
\text { in shoot dry matter yield } \\
\text { (10 wk) }\end{array}$ \\
\hline $\begin{array}{l}\text { Noguera et al., } \\
2010\end{array}$ & Colombia & inceptisol & rice & $\begin{array}{l}\text { charcoal (wood) }(0.2 \\
\text { and } 0.5 \% \mathrm{w} / \mathrm{w})(\mathrm{lab} \\
\text { produced) }\end{array}$ & +30 to $+200 \%$ \\
\hline Nutman, 1952 & U.K. & test tube/no soil & clover & $\begin{array}{l}\text { charcoal }(0.5-2 \% \\
\text { w/w) }\end{array}$ & $\begin{array}{l}\text { beneficial increases in } \\
\text { nodulation; does not } \\
\text { occur if charcoal is ashed }\end{array}$ \\
\hline O'Toole, 2010 & Norway & Fluvic cambisol & $\begin{array}{l}\text { rye grass (Lolium } \\
\text { perenne } L)\end{array}$ & $\begin{array}{l}\text { wheat-straw biochar } \\
\text { (up to } 30 \% \mathrm{w} / \mathrm{w} \text { ) }\end{array}$ & $\begin{array}{l}\text { no effect with fertilizers; } \\
\text { without or low } \\
\text { fertilization: negative } \\
\text { yield impacts }\end{array}$ \\
\hline $\begin{array}{l}\text { Oguntunde et al., } \\
2004\end{array}$ & Ghana & compared charcoal kiln soils & corn & $\begin{array}{l}\text { compared wood } \\
\text { charcoal kiln soils } \\
\text { with non kiln soils } \\
\text { (unknown rates) }\end{array}$ & $\begin{array}{l}\text { +90\%; observed } \\
\text { differences were not fully } \\
\text { explainable by nutrient } \\
\text { availability }\end{array}$ \\
\hline Rajkovich, 2010 & U.S. & silt loam and loam & corn & $\begin{array}{l}\text { variety of feedstocks } \\
\text { examined; food } \\
\text { wastes, paper mill } \\
\text { wastes, wood, and } \\
\text { manures at various } \\
\text { temperatures }(0.2, \\
0.5,2 \text {, and } 7 \% \mathrm{w} / \mathrm{w})\end{array}$ & $\begin{array}{l}\text { decreased biomass seen } \\
\text { in about one third of the } \\
\text { tested mixtures: food } \\
\text { wastes biochar ( }-18 \text { to } \\
-85 \%), \text { papermill biochar } \\
(-85 \%),+17 \% \text { increase in } \\
\text { poultry manure biochars } \\
(+17 \%)\end{array}$ \\
\hline $\begin{array}{l}\text { Rondon et al., } \\
2007\end{array}$ & Columbia & clay-loam oxisol (Typic Haplustox) & beans ( $P$. vulgaris L.) & $\begin{array}{l}\text { kiln charcoal }(0,30 \text {, } \\
60 \text {, and } 90 \mathrm{~g} \mathrm{~kg}^{-1} \\
\text { soil) }\end{array}$ & $\begin{array}{l}46 \%(\leq 60) ;>90 \text { resulted in } \\
\text { yield decrease }\end{array}$ \\
\hline $\begin{array}{l}\text { Rutto and } \\
\text { Mizutani, } 2006\end{array}$ & China & growing media & peach & $\begin{array}{l}\text { activated charcoal } \\
\text { (unknown) }\end{array}$ & no differences \\
\hline
\end{tabular}


Table 3. Continued.

\begin{tabular}{|c|c|c|c|c|c|}
\hline Reference & Country & Soil type & Crop & Addition/rate & $\begin{array}{c}\text { Yield results } \\
\text { (compared with control) }\end{array}$ \\
\hline $\begin{array}{l}\text { Solaiman et al., } \\
2010\end{array}$ & Australia & sandy clay loam (field) & wheat (Triticum aestivum) & $\begin{array}{l}\text { biochar }(0,1.5,3.0, \\
\left.\text { and } 6 \mathrm{t} \mathrm{ha}^{-1}\right)\end{array}$ & $\begin{array}{l}\text { increased yields at low } \\
\text { fertilizer rates; no } \\
\text { significant differences at } \\
\text { recommended levels }\end{array}$ \\
\hline $\begin{array}{l}\text { Sorensen et al., } \\
\text { unpublished }\end{array}$ & U.S. & $\begin{array}{l}\text { Greenville fine sandy loam (fine, } \\
\text { kaolinitic, thermic Rhodic } \\
\text { Kandiudults) }\end{array}$ & cotton & $\begin{array}{l}\text { fast pyrolysis } \\
\text { hardwood }(22,45, \\
89, \text { and } 135 \mathrm{Mg} \\
\left.\mathrm{ha}^{-1}\right)\end{array}$ & no difference (year 1) \\
\hline \multirow[t]{5}{*}{$\begin{array}{l}\text { Spokas, } \\
\text { unpublished }\end{array}$} & U.S. & $\begin{array}{l}\text { Waukegan silt loam (fine-silty over } \\
\text { skeletal mixed, super active, mesic } \\
\text { Typic Hapludoll) Field plots }\end{array}$ & corn & $\begin{array}{l}\text { fast pyrolysis sawdust } \\
\text { biochar }(22,460 \mathrm{~kg} \\
\left.\text { ha }^{-1}\right)\end{array}$ & no difference (year 1) \\
\hline & & & corn & $\begin{array}{l}\text { slow pyrolysis } \\
\text { woodwaste }(22,460 \\
\left.\mathrm{kg} \mathrm{ha}^{-1}\right)\end{array}$ & no difference (year 1) \\
\hline & & & corn & $\begin{array}{l}\text { slow pyrolysis wood } \\
\text { pellet biochar } \\
\left(22,460 \mathrm{~kg} \mathrm{ha}^{-1}\right)\end{array}$ & no difference (year 1) \\
\hline & & $\begin{array}{l}\text { potting soil mix (greenhouse } \\
\text { experiment) }\end{array}$ & lettuce, spinach, radish & $\begin{array}{l}\text { fast pyrolysis } \\
\text { macadamia nut } \\
(10 \% \mathrm{w} / \mathrm{w})\end{array}$ & \multirow{2}{*}{$\begin{array}{l}\text { significant decrease } \\
\text { in growth rate, } \\
\text { germination timing, and } \\
\text { biomass production } \\
\text { observed with biochar }\end{array}$} \\
\hline & & $\begin{array}{l}\text { potting soil mix (greenhouse } \\
\text { experiment) }\end{array}$ & lettuce, spinach, radish & $\begin{array}{l}\text { slow pyrolysis wood } \\
\text { pellet biochar }(10 \% \\
\text { w/w) }\end{array}$ & \\
\hline $\begin{array}{l}\text { Steiner et al., } \\
2007\end{array}$ & Brazil & Xanthic Ferralsol (weathered) & $\begin{array}{l}\text { rice (Oryza sativa L.) and } \\
\text { sorghum (Sorghum bicolor } \\
\text { L.) rotation }\end{array}$ & $\begin{array}{l}\text { charcoal (forest } \\
\text { wood) }\left(11 \mathrm{Mg} \mathrm{ha}^{-1}\right. \\
\text { charcoal (reference } \\
\text { has } 11 \mathrm{mg} \mathrm{ha}^{-1}, \\
\text { assumed Mg) }\end{array}$ & $\begin{array}{l}+170 \% \text { with fertilizer; } \\
\text { charcoal additions } \\
\text { alone did not increase } \\
\text { production }\end{array}$ \\
\hline $\begin{array}{l}\text { Suhardi et al., } \\
2006\end{array}$ & Indonesia & Bukit Suharto experiment field & Shorea leprosula & $\begin{array}{l}\text { charcoal }+ \text { fertilizer } \\
(0-100 \mathrm{~g} \text { of charcoal } \\
\text { per plot })\end{array}$ & $\begin{array}{l}\text { no impact on height, } \\
\text { plant diameter or } \\
\text { mycorrhizal formation }\end{array}$ \\
\hline \multirow[t]{4}{*}{ Tagoe et al., 2008} & Africa & & soybean & chicken manure BC & $+41 \%$ \\
\hline & & & cowpea & chicken manure $\mathrm{BC}$ & $+146 \%$ \\
\hline & & & soybean & $\begin{array}{l}\text { municipal organic } \\
\text { waste biochar }\end{array}$ & $+20 \%$ \\
\hline & & & cowpea & $\begin{array}{l}\text { municipal organic } \\
\text { waste biochar }\end{array}$ & $+59 \%$ \\
\hline $\begin{array}{l}\text { Topoliantz et al., } \\
2005\end{array}$ & $\begin{array}{l}\text { French } \\
\text { Guiana }\end{array}$ & Oxisol & $\begin{array}{l}\text { bean ( } V \text {. unguiculata } \\
\text { sesquipedalis) }\end{array}$ & charcoal (wood) & $\begin{array}{l}\text { increased yield with green } \\
\text { manure (manioc peels) }\end{array}$ \\
\hline Vaccari et al., 2011 & Italy & silty loam & wheat & wood charcoal & $\begin{array}{l}20-40 \% \text { increase (only } \\
\text { significant at } P=0.10 \text { ) }\end{array}$ \\
\hline \multirow[t]{3}{*}{$\begin{array}{l}\text { Vantsis and Bond, } \\
1950\end{array}$} & U.K. & sand & clover & $\begin{array}{l}\text { wood charcoal }(0.5- \\
2 \% \mathrm{w} / \mathrm{w})\end{array}$ & $\begin{array}{l}\text { increases in dry weight } \\
\text { and nitrogen fixation }\end{array}$ \\
\hline & & & clover & $\begin{array}{l}\text { animal charcoal } \\
\text { (bone) }(0.5-2 \% \\
\text { w/w) }\end{array}$ & inhibition of growth \\
\hline & & & clover & $\begin{array}{l}\text { activated charcoal } \\
(0.5-2 \% \mathrm{w} / \mathrm{w})\end{array}$ & $\begin{array}{l}\text { increases in dry weight \& } \\
\mathrm{N} \text { fixation }\end{array}$ \\
\hline $\begin{array}{l}\text { Wang and Huang, } \\
1976\end{array}$ & Taiwan & culture media & various & $\begin{array}{l}\text { activated charcoal (3 } \\
\left.\mathrm{g} \mathrm{L}^{-1}\right)\end{array}$ & $\begin{array}{l}\text { improves growth by } \\
\text { sorbing toxic metabolites }\end{array}$ \\
\hline \multirow[t]{3}{*}{ Yamato et al., 2006} & Indonesia & Indonesia farmland soils (weathered) & corn & $\begin{array}{l}\text { Acacia mangium (kiln } \\
\text { bark charcoal), } 10 \\
\mathrm{~L} \mathrm{~m}^{-2}\end{array}$ & $\begin{array}{l}\text { site A: }+190 \% \text {; site B: no } \\
\text { difference }\end{array}$ \\
\hline & & Indonesia farmland soils (weathered) & cowpea & $\begin{array}{l}\text { A. mangium (kiln bark } \\
\text { charcoal), } 10 \mathrm{~L} \mathrm{~m}^{-2}\end{array}$ & no differences \\
\hline & & Indonesia farmland soils (weathered) & peanut & $\begin{array}{l}\text { A. mangium (kiln bark } \\
\text { charcoal), } 10 \mathrm{~L} \mathrm{~m}^{-2}\end{array}$ & $\begin{array}{l}\text { site } A:+100 \% \text {; site B: no } \\
\text { difference }\end{array}$ \\
\hline Zhang et al. 2011 & China & Entic halpudept & rice & $\begin{array}{l}\text { wheat straw biochar } \\
\left(10 \text { and } 40 \mathrm{tha}^{-1}\right)\end{array}$ & $8-14 \%$ increase \\
\hline
\end{tabular}

† Black carbon.

Other explanations for biochar's crop yield impact have ranged from $\mathrm{N}$ immobilization leading to decreased $\mathrm{N}$ availability due to the high $\mathrm{C} / \mathrm{N}$ biochar ratios (Rondon et al., 2007), liming effects of the biochar (Verheijen et al., 2009), reduced plant availability of macronutrients due to $\mathrm{pH}$ altera- tions (Hiradate et al., 2007; Makoto et al., 2010), and direct sorption of soil nutrients (Asai et al., 2009). Asai et al. (2009) tested the influence of biochar additions on a variety of soil types at 10 different locations and observed yield increases in soils with low $\mathrm{P}$ availability and improved plant response to 
additional fertilizers with biochar additions. However, these findings are not universal; even fertilizer plus biochar additions have resulted in suppressed yields in some cases (e.g., Table 3). Therefore, soil nutrient status alone is not sufficient to explain all the crop responses observed but could be important after biochar amendments to weathered and low $\mathrm{N}$ - and P-containing soils due to fertilization (Table 2).

There are additional potential mechanisms responsible for biochar's effect on agronomic yield. Studies have shown altered rates and timing of seed germination as a function of biochar additions (e.g., de Keijzer and Hermann, 1966; Rillig et al., 2010). Differences in germination and consequentially plant emergence could influence plant growth and yield due to the timing of precipitation and accumulation of thermal time. In other words, plant seeds that are simultaneously sown in biochar-amended and non-biochar-amended soils that emerge at different times are also temporally equivalent to a varying planting date. Differences in planting date in field plots have been observed to affect plant growth and yield due to the timing of precipitation and accumulation of growing degree days (Egli and Bruening, 1992).

Biochar can also sorb, release, or catalyze transformations of compounds that affect plant and microbial growth. Black carbon has been observed to catalyze abiotic transformations of nitrogen-containing compounds (i.e., ammonia, nitric oxide, nitrate, or nitrous oxide) even at ambient conditions (Chang and Novakov, 1975; DeGroot et al., 1991; Aarna and Suuberg, 1997), particularly linked to ammonium formation from other $\mathrm{N}$ forms (Chang and Novakov, 1975). Black carbon has also been observed to catalyze the transformation of sulfur compounds, leading to the formation of sulfates (Novakov et al., 1974). Despite the fact that some of these studies investigated nonbiomass $\mathrm{BC}$ forms, biochar is $\mathrm{BC}$ and could possess chemistries similar to nonbiomass source $\mathrm{BC}$ as a function of production and processing conditions (Spokas, 2010). In addition to direct abiotic transformation of soil nutrients, the sorption of soil inhibitory chemicals by BC was hypothesized to be responsible for alterations in clover nodulation (Turner, 1955). The role of volatile organics in soil microbial and plant signaling is an emerging field (Insam and Seewald, 2010). In some cases, these VOCs may be sorbed by biochar particles (Turner, 1955; Warnock et al., 2007), whereas at other times VOC may be emitted from biochars (Spokas et al., 2010). This release or sorption of VOCs may cause plant allelopathic reactions and may inhibit or stimulate microbial functionality and positive or negative plant effects (Deenik et al., 2010; Graber et al., 2010). However, these chemical effects would be dependent on soil, microbial, plant, and biochar properties. Therefore, this role of biochar sorbing or releasing inhibitory chemicals could explain seemingly contradictory results because the effect would be a function of the respective concentration thresholds for the specific microbe or plant. However, the commonality of this hypothesis is lacking because characterization of the sorbed compounds is not a typical analysis conducted on biochar.

Biochar-induced yield improvements are further complicated by the occasional delayed response, with negative or no impact in the initial year followed by yield increases of varying degrees in subsequent years (Kulmatiski and Beard, 2006; Gaskin et al., 2010; Major et al., 2010b). These delayed responses are hypothesized due to aging of the biochar (e.g., oxidation or other chemical alteration) (Puri et al., 1958; Allardice, 1966; Cheng et al., 2006; Singh et al., 2010b). Chemical or thermal biochar activation drastically alters the surface chemistry (Azargohar and Dalai, 2006, 2008; Nuithitikul et al., 2010). Chemisorption of oxygen by biochar also alters the surface chemistry (Puri et al., 1958) and microbial degradability (Cheng et al., 2008), which could affect biochar nutrient availability. These abiotic chemisorption reactions occur at ambient conditions (Itay et al., 1989), which have only received limited attention in the biochar literature (Cheng et al., 2006; Spokas et al., 2009; Zimmerman, 2010; Jones et al., 2011). These postprocessing reactions can drastically alter the biochar and resulting observed impacts, which again highlights the need for documenting postproduction handling and storage of biochar.

The influence of biochar on soil fertility may be positive or negative depending on the quality and rate of biochar applied, with some uncertainty as to the exact mechanisms. As with soil application of other byproducts (Sumner, 2000; Ippolito et al., 2011), an application of high-nutrient biochar that exceeds recommended fertilization rates may unbalance soil nutrient levels, produce little improvement in soil nutrient retention, and increase nutrient leaching potentials. Some biochar nutrients are leachable despite the observations of nutrient sorption (Ding et al., 2010). Although control soils had no detectable soluble $\mathrm{N}$ or $\mathrm{P}$ in the leachate, the first leachate collected from soils treated with poultry litter biochar contained 3 and $8 \mu \mathrm{g}$ $\mathrm{mL}^{-1}$ of $\mathrm{NH}_{4}-\mathrm{N}$ and $\mathrm{NO}_{3}-\mathrm{N}$, respectively, and $36 \mu \mathrm{g} \mathrm{mL}^{-1}$ dissolved P concentrations (Novak et al., 2009b). The addition of hardwood charcoal to a typical Midwestern agricultural soil (Hapludoll) in a laboratory column study substantially reduced $\mathrm{P}$ and $\mathrm{N}$ nutrient leaching from a subsequent manure addition compared with controls, with greater reductions correlated with increasing charcoal rates (Laird et al., 2010a). However, the amounts of $\mathrm{K}, \mathrm{Mg}, \mathrm{Zn}, \mathrm{Ca}$, and total $\mathrm{N}$ leached from the columns only receiving charcoal were greater than the control columns, hence the conclusion that some charcoal nutrients are partially leachable (Laird et al., 2010b).

Soil nutrient improvements may take some time to be observed. One could envision a delay if the particular element is enclosed in a chemical ring structure because the kinetics of surface functional group oxidation and cleavage of ring structures would be rate limiting (Glaser et al., 2002; Liang et al., 2006; Yao et al., 2010). However, a majority of the existing studies have been limited to less than $3 \mathrm{yr}$, which may not be enough time for the soil nutrient cycle to be affected.

In conclusion, plant responses to biochar additions are the net result of production (e.g., feedstock and pyrolysis conditions) and postproduction (storage or activation) conditions. These processes can infer potentially unique properties to each batch of biochar, even from the same pyrolysis unit and biomass feedstock. The mechanisms resulting in negative plant impacts need to be fully understood due to the significant plant biomass reductions (35-87\% of nonamended soil controls) that have been cited as a function of feedstock and production temperatures (Table 3). However, production temperature alone cannot describe the variability in laboratory biochar microbial impact assessments (Spokas, 2010). 
Due to the lack of universal properties and characterization reported in biochar studies, full elucidation of the responsible processes from literature studies is unfeasible and requires additional detailed studies (Lehmann et al., 2011), particularly documenting biochar production and postproduction handling. Based on the available data, the processes by which biochar improves plant growth and yield are debatable, but this knowledge is critical to fully optimize biochar for agronomic purposes.

\section{Biochar Economics}

Assessing the economic feasibility of biochar requires evaluation of the entire system, which starts with feedstock production and preparation, transportation to the pyrolysis unit, net energy consumption or production of the pyrolysis process itself, storage, and biochar utilization, including transportation and application costs. Some of these costs could be offset by the other products that are typically coproduced with biochar and by potential sustained biochar benefits. At the pyrolysis facility that targets energy production, biochar represents a loss in energy production, which is a loss of revenue. As an aside, biochar can be used as an energy source (Boateng, 2007; Abdullah and Wu, 2009; Abdullah et al., 2010). However, this use of biochar as a fuel would not fall under the definition of biochar because there is no carbon sequestration. Direct energy use of biochar will be a competing use to carbon sequestration.

Increasing soil aggregation, water infiltration, and waterholding capacity may also reduce irrigation costs, especially in semiarid environments. Drought and subsequent crop stress is common in the arid western United States, and thus irrigation is commonly used to meet crop water demands. Soils in the arid to semiarid regions of the U.S. Pacific Northwest are typically fine-textured Aridisols and Mollisols that inherently have a high water-storage capacity. However, irrigation water quickly evaporates; consequently, the length of time that soil water is available for crops after irrigation or precipitation is of paramount concern. In a laboratory column leaching study, Brockhoff et al. (2010) observed an increase of $370 \%$ in waterholding capacity under gravity drainage after a $25 \%$ (v/v) biochar addition to sand. An increase in soil moisture storage due to biochar application could be appealing to crop producers in the semiarid to arid regions of the United States and in other drought-plagued regions around the world with limitated irrigation water availability. For the agricultural producer, the cost of biochar must be low enough that the benefits of soil application exceed the total cost of biochar application or the cost of applying additional irrigation water.

Select economic analyses have been conducted looking at the entire system. Roberts et al. (2010) conducted a life cycle assessment of biochar systems using corn stover, switchgrass, or yard wastes as feedstocks for greenhouse gas (GHG) offset values of $\$ 20 \mathrm{Mg}^{-1} \mathrm{CO}_{2}$ equivalents $\left(\mathrm{CO}_{2} \mathrm{e}\right)$ and $\$ 80 \mathrm{Mg}^{-1} \mathrm{CO}_{2}$ e. Only the yard waste feedstock could profitably be used for pyrolysis with $\$ 20 \mathrm{Mg}^{-1} \mathrm{CO}_{2}$ e. However, all feedstocks were profitable at $\$ 80 \mathrm{Mg}^{-1} \mathrm{CO}_{2} \mathrm{e}$, with the exception of one switchgrass scenario, where switchgrass production led to large indirect land use changes that negated the GHG benefits. This analysis assumed that $80 \%$ of the $\mathrm{C}$ in biochar was stable, with the remaining
$20 \%$ released as $\mathrm{CO}_{2}$ within the first few years of application (Roberts et al., 2010). This assumption is questionable given the fact that biochar is not a singular homogeneous product and that the degradability is directly tied to the overall chemistry (Spokas, 2010), which varies greatly as a function of biochar type (Zimmerman, 2010) as well as the variability of the biochar itself (Hedges et al., 2000). The analysis also assumed that there were no yield benefits to biochar application but that $\mathrm{N}, \mathrm{P}$, and $\mathrm{K}$ fertilizer use was reduced by $7.2 \%$ with biochar application and $\mathrm{N}_{2} \mathrm{O}$ emissions were reduced by $50 \%$. These may or may not be realistic assumptions due to the complex interaction of biochar and soil $\mathrm{N}$-cycling and due to variations in the responses due to biochar and soil types as previously described.

McCarl et al. (2009) showed that both fast and slow pyrolysis production of biochar from corn stover was not economically feasible. The results were sensitive to pyrolysis facility costs, GHG offset price, energy prices, and impacts of biochar on crop yields. The analysis showed that pyrolysis would be profitable with GHG offset prices of more than $\$ 58 \mathrm{Mg}^{-1} \mathrm{CO}_{2}$ e or $\$ 71 \mathrm{Mg}^{-1} \mathrm{CO}_{2}$ e for fast or slow pyrolysis, respectively. The analysis also showed that pyrolysis would be profitable for biochar prices greater than $\$ 450 \mathrm{Mg}^{-1}$ or $\$ 246$ $\mathrm{Mg}^{-1}$ for fast or slow pyrolysis, respectively. These prices were substantially greater than the 2008 biochar energy value from combustion $\left(\$ 55 \mathrm{Mg}^{-1}\right)$ or soil application value $\left(\$ 47 \mathrm{Mg}^{-1}\right)$ (McCarl et al., 2009). The soil application value was based on the assumption of a persistent $5 \%$ maize yield increase and annual input cost savings of $\$ 73 \mathrm{ha}^{-1}$ due to reductions in need for nutrients, lime, and seed but did not include the value of any GHG offsets. The estimated soil application value might be conservative if the short-term $12 \%$ yield improvement indicated in the Verheijen et al. (2009) metaanalysis was obtained with maize production and was present for many years after biochar application. The magnitude and persistence of yield benefits are key to understanding the potential economic value of biochar application.

Granatstein et al. (2009) conducted an economic analysis on pyrolyzing biomass from forest thinning. Results showed that the lowest breakeven cost for a facility producing biochar was $\$ 87 \mathrm{Mg}^{-1}$ biochar for a stationary facility and up to $\$ 1,742 \mathrm{Mg}^{-1}$ biochar for a mobile unit. The potential impacts of GHG offsets were not included in the analysis. In a related study, Galinato et al. (2010) analyzed the profitability of applying biochar instead of agricultural lime to winter wheat fields in eastern Washington. The only crop production impact of the biochar included in the analysis was the effect on crop yields through changing soil $\mathrm{pH}$. Without GHG offset payments, analysis showed that the biochar price would need to be less than $\$ 4.34 \mathrm{Mg}^{-1}$ for biochar application to be more profitable than lime. The breakeven biochar price for the farmer, or the price farmers would be willing to pay, will increase with increasing GHG offset payments, assuming the GHG offset is paid to the farmer. For example, given a GHG offset payment of $\$ 31 \mathrm{Mg}^{-1} \mathrm{CO}_{2} \mathrm{e}$, biochar application is more profitable than lime application for biochar prices less than $\$ 96 \mathrm{Mg}^{-1}$ (Galinato et al., 2010). At this level, the agricultural value exceeds the lowest breakeven cost for a stationary facility, indicating the potential for profitable production of biochar. 
Galinato et al. (2010) also alluded to an important aspect of evaluating the value of biochar for field application. Comparisons must be made with the next most profitable alternative, not simply a no-biochar alternative. The breakeven price of biochar using this comparison was always higher than the price relative to the alternative with agricultural lime because this was the most profitable practice. Therefore, omitting relevant biochar alternatives from the analysis can falsely skew the predicted value of biochar.

Focusing on the value of $\mathrm{CO}_{2}$ reductions, Gaunt and Lehmann (2008) determined that biochar application to agricultural land would provide greater GHG emission reductions than using biochar for electricity generation. However, the costs of achieving the additional GHG emission through soil application ranged from 9 to $\$ 16 \mathrm{Mg}^{-1} \mathrm{CO}_{2} \mathrm{e}$. This cost was based on the value of lost electricity generation and did not include the value of any agricultural production benefits. The calculated GHG emission reductions with biochar addition did, however, assume a fertilizer reduction of $10 \%$, which would result in a $50 \%$ reduction in $\mathrm{N}_{2} \mathrm{O}$ emissions, and that the effect of biochar would remain for $10 \mathrm{yr}$ after application.

A combined biochar life cycle and cost-benefit analysis was conducted by Tejerina (2010). This analysis used monetary, energy, and GHG balances (assuming a 10\% fertilizer savings and a 5\% yield increase with biochar) in the context of a developing country under three different management scenarios: (i) biochar for soil application, (ii) bio-coal production, or (iii) current management (residue left on the field). The results showed corn stover used for biochar production and soil application as providing the greatest net energy and positive revenue potential over bio-coal production or leaving the residue in the field. However, the results for a developed country could be drastically different due to economic differences in the assumed costs for labor, machinery, transportation, and energy.

In each of these above cases, the value of biochar for field application was sensitive to uncertainties associated with the annual value of benefits (or costs) that result from soil application and the duration of these benefits (or costs). Annual benefits may accrue if a biochar application increased yields or reduced production costs. However, our ability to extract a defensible yield improvement prediction from the existing data is hampered by insufficient biochar characterization data coupled to the lack of uniform yield responses (Table 3). Woodbased biochars, which typically increase agronomic yields, are the biochars with the lowest nutrient contents (Table 2). The GHG offset payments associated with avoided emissions due to reduced fertilizer, pesticide, or other inputs use may also be included as annual benefits once the duration of these benefits is known. Long-term, small annual biochar benefits can produce high value to farmers over time. This means that biochar application is economically feasible at the field-scale if it is available to farmers at a cost less than a break-even value. However, the amount farmers are willing to invest in biochar applications will be directly related to the certainty of attaining the degree and persistence of predicted annual benefits. On the other hand, any potential persistent negative cost effects can substantially reduce the value of biochar to the farmer.

The value of GHG offset payments is typically based on permanent GHG offsets, so the soil carbon sequestration value of biochar application would have to be included as a one-time benefit in the above analysis and not as a repeating annual benefit. In addition, the value would be reduced if sequestration was not long-term. Furthermore, the lack of standardization in the accounting of renewable energy system GHG assessments creates systematic biases that can lead to over- or underestimation of the net GHG impacts (Whitman and Lehmann, 2011).

\section{Potential Specialized Markets}

The possibility of niche specialized uses of biochar could substantially improve the economic picture for biochar utilization, particularly engineering "designer biochars" for improving a specific soil deficiency (Novak and Busscher, 2011). For example, there have been efforts at impregnating biochar with inorganic fertilizers to serve as a slow-release fertilizer (Khan et al., 2008) and to provide localized crop protection from herbicides, pesticides, and other chemicals (Fink, 1934; Linscott and Hagin, 1967). Biochar could be blended with compost (Rosenfeld, 2001; Rosenfeld and Henry, 2001; Steiner et al., 2010), which could increase biochar's value, particularly if biochar application for bedding plants is beneficial (Kadota and Niimi, 2004).

Biochar might be useful for controlling selected invasive or nuisance species of animals (Mason and Clark, 1994, 1995) and plants (Kulmatiski and Beard, 2006). Biochar may offer the potential as a means for contaminated site cleanup (Cao and Harris, 2010). An example could be reducing soil copper contamination resulting from irrigation with water from spent copper sulfate $\left(\mathrm{CuSO}_{4}\right)$ dairy hoof bath lagoons (Ippolito et al., 2012) or as a potential remediation tool for acid mine soils (Novak and Busscher, 2011).

Biochar use does not have to be limited to soil application. Biochar could be suitable as a precursor to generate activated carbon, which is commonly used in industrial filtration processes (Azargohar and Dalai, 2006). However, the production of activated charcoal requires extra conditioning steps, which reduces the economic return (Lussier et al., 1994). Several studies have examined biochar and activated biochar use in municipal wastewater treatment ( $\mathrm{Ng}$ et al., 2002, 2003; Bansode et al., 2004; Lima and Marshall, 2009), in mercury removal from flue gas (Klasson et al., 2010), and in other water filtering systems (van Duck and van de Voorde, 1984). However, for biochar to be used for potable water filtration, the potential for bacterial growth and organic contaminates on the biochar needs to be further investigated (Wallis et al., 1974). Other potential uses of biochar include use as a nutrient recovery agent (Streubel et al., 2010), as an additive for reducing the bioavailability and mobility of toxic trace metals (Beesley and Marmiroli, 2011; Uchimiya et al., 2011), as a contaminant mitigation agent (Beesley et al., 2010), or as a material for iron and steel production (de Beer et al., 1998). Potential markets in laboratory settings exist for biochar, including solid-phase microextraction fibers (Wan et al., 1994), electroanalytical chemistry electrodes (Tavares and Barbeira, 2008), or biochar-based DNA hybridization biosensors (Wang and Kawde, 2001). Spent biochar could be examined for uses in landfill covers for odor and bird control (Mason and Clark, 1994). Furthermore, the potential use of biochar as a sorbent media 
for scrubbing $\mathrm{CO}_{2}$ from fossil fuel stack emissions warrants additional research because this could offer additional carbon sequestration potential for biochar (Mercedes Maroto-Valer et al., 2008). All of the above applications preserve the carbon sequestration potential. In addition to direct applications, the use of biochar as a green product label also offers potential targeted economic advantages (Yoder and Galinato, 2009).

We acknowledge that biochar is expensive as a carbon sequestration agent or as a soil supplement for crop yield improvements. However, the high production cost for biochar could be offset if these specialty or boutique markets are more fully developed. The key is to diversify biochar applications to other sectors, which could result in reduced costs for production.

\section{Future Needs}

Just as economics caused the shift from biomass to fossil fuels in the early 1920s, civilization is currently at the cusp where environmental stewardship is returning the pendulum back to biomass as the source for human's energy, chemical, and agronomic needs. Given this return to biomass, there are several factors that should be considered as we move forward:

- There is a fundamental need to recognize that biochar does not refer to one singular product; it refers to the range of potential products produced for carbon sequestration purposes.

- There is a vital need to fully document the production style, biomass conditions, and pyrolysis conditions of the biochar production, but almost equally important is the time since biochar production, postproduction handling, and storage conditions. This is especially evident given the importance of the surface chemistry on overall biochar chemistry and resulting environmental interactions.

- As seen in several aspects of this review, the lack of adequate data has limited the ability to use the historic data in meta-analyses to elucidate driving variables. This is due to the lack of consistency in biochar characterization and documentation, which is partially a result of the incomplete understanding of the mechanisms responsible for agronomic yield improvements. In other words, what properties do we want biochar to possess?

- The studies using traditional kilns and production systems without industrial controls are further suspect because there is no documentation on production parameters (e.g., pyrolysis temperature, oxygen status, etc.) or repeatability of the biochar production style.

- There is also a need to overcome the terminology and definition hurdles and to separate the soil impacts of biochar additions from the biochar itself. This is needed to continue the development of biochar as a stable carbon form that could be considered for carbon sequestration markets. The potential end uses for biochar are virtually limitless, and these specialized market niches potentially could increase the economic value of biochar.

- There is a need for the creation of an international pool of well characterized biochars for systematic research (Lehmann et al., 2011). Furthermore, these biochars need to come from a variety of sources with well documented production systems to achieve a crosssectional sampling required for such an effort. This effort could be coordinated by the American Society of Agronomy's Biochar Community (https://www. agronomy.org/membership/communities/biocharagronomic-and-environmental-uses).

- We need to better understand how biochar's production conditions influence its quality and subsequent effects on the soil-plant systems, which would lead to more accurate guidance for biochar amendment management.

A tipping point is being approached where global demand for food will exceed production. Despite the current forecasted economics, the fact that biochar has the potential to improve soil quality and provide benefits to nonproductive and degraded soils justifies continued research efforts into biochar's soil quality impacts. Continued research can optimize biochar production for the overall health and fertility of our soil resources, which represent our most vital asset in the current bioenergy renaissance and are the fundamental foundation for food security.

\section{Acknowledgments}

This manuscript is a joint product of the United States Department of Agriculture-Agricultural Research Service (USDA-ARS) Biochar and Pyrolysis Initiative and GRACEnet programs. The USDA-ARS has established an integrated long-term research effort examining pyrolysis and biochar research at multiple locations across the United States, with various coordinated field and laboratory components (USDA Biochar and Pyrolysis Initiative). Some of the results from these USDA-ARS studies were included here. In addition, the authors would like to acknowledge the partial funding from the Minnesota Corn Growers Association/Minnesota Corn Research Production Council and the Minnesota Department of Agriculture Specialty Crop Block Grant program. The authors also acknowledge the assistance of the anonymous reviewers who provided helpful suggestions on improvements to the manuscript.

\section{References}

Aarna, I., and E.M. Suuberg. 1997. A review of the kinetics of the nitric oxide-carbon reaction. Fuel 76:475-491. doi:10.1016/ S0016-2361(96)00212-8

Abdullah, H., and H.W. Wu. 2009. Biochar as a fuel: 1. Properties and grindability of biochars produced from the pyrolysis of Mallee wood under slowheating conditions. Energy Fuels 23:4174-4181. doi:10.1021/ef900494t

Abdullah, H., K.A. Mediaswanti, and H.W. Wu. 2010. Biochar as a fuel: 2. Significant differences in fuel quality and ash properties of biochars from various biomass components of Mallee trees. Energy Fuels 24:19721979. doi:10.1021/ef901435f

Aiman, S., and J.F. Stubington. 1993. The pyrolysis kinetics of bagasse at low heating rates. Biomass Bioenergy 5:113-120. doi:10.1016/0961-9534(93)90092-I

Alén, R., E. Kuoppala, and P. Oesch. 1996. Formation of the main degradation compound groups from wood and its components during pyrolysis. J. Anal. Appl. Pyrolysis 36:137-148. doi:10.1016/0165-2370(96)00932-1

Allardice, D.J. 1966. The adsorption of oxygen on brown coal char. Carbon 4:255-262. doi:10.1016/0008-6223(66)90087-X

Anonymous. 1840. Peat and charcoal. J. Franklin Inst. 29:203-204.

Antal, M.J., and M. Grønli. 2003. The art, science, and technology of charcoal production. Ind. Eng. Chem. Res. 42:1619-1640. doi:10.1021/ ie0207919

Apaydin-Varol, E., E. Pütün, and A.E. Pütün. 2007. Slow pyrolysis of pistachio shell. Fuel 86:1892-1899. doi:10.1016/j.fuel.2006.11.041

Asai, H., B.K. Samson, H.M. Stephan, K. Songyikhangsuthor, K. Homma, Y. Kiyono, Y. Inoue, T. Shiraiwa, and T. Horie. 2009. Biochar amendment 
techniques for upland rice production in Northern Laos: 1. Soil physical properties, leaf SPAD and grain yield. Field Crops Res. 111:81-84. doi:10.1016/j.fcr.2008.10.008

Atkinson, C., J. Fitzgerald, and N. Hipps. 2010. Potential mechanisms for achieving agricultural benefits from biochar application to temperate soils: A review. Plant Soil 337:1-18. doi:10.1007/s11104-010-0464-5

Azargohar, R., and A.K. Dalai. 2006. Biochar as a precursor of activated carbon. Appl. Biochem. Biotechnol. 131:762-773. doi:10.1385/ ABAB:131:1:762

Azargohar, R., and A.K. Dalai. 2008. Steam and KOH activation of biochar: Experimental and modeling studies. Microporous Mesoporous Mater. 110:413-421. doi:10.1016/j.micromeso.2007.06.047

Ball, P.N., M.D. MacKenzie, T.H. DeLuca, and W.E.H. Montana. 2010. Wildfire and charcoal enhance nitrification and ammonium-oxidizing bacterial abundance in dry Montane forest soils. J. Environ. Qual. 39:1243-1253. doi:10.2134/jeq2009.0082

Bansode, R.R., J.N. Losso, W.E. Marshall, R.M. Rao, and R.J. Portier. 2004. Pecan shell-based granular activated carbon for treatment of chemical oxygen demand (COD) in municipal wastewater. Bioresour. Technol. 94:129-135. doi:10.1016/j.biortech.2003.12.009

Bapat, H.D., and S.E. Manahan. 1998. Chemchar gasification of hazardous wastes and mixed wastes on a biochar matrix. Abstr. Papers Am. Chem. Soc. 215:008-ENVR.

Baumann, B. 1960. The botanical aspects of ancient Egyptian embalming and burial. Econ. Bot. 14:84-104. doi:10.1007/BF02859368

Beesley, L., and M. Marmiroli. 2011. The immobilisation and retention of soluble arsenic, cadmium and zinc by biochar. Environ. Pollut. 159:474480. doi:10.1016/j.envpol.2010.10.016

Beesley, L., E. Moreno-Jimenez, and J.L. Gomez-Eyles. 2010. Effects of biochar and greenwaste compost amendments on mobility, bioavailability and toxicity of inorganic and organic contaminants in a multielement polluted soil. Environ. Pollut. 158:2282-2287. doi:10.1016/j. envpol.2010.02.003

Boateng, A.A. 2007. Characterization and thermal conversion of charcoal derived from fluidized-bed fast pyrolysis oil production of switchgrass. Ind. Eng. Chem. Res. 46:8857-8862. doi:10.1021/ie0710541

Boateng, A.A., C.A. Mullen, and N.M. Goldberg. 2010a. Producing stable pyrolysis liquids from the oil-seed presscakes of mustard family plants: Pennycress (Thlaspi arvense L.) and Camelina (Camelina sativa). Energy Fuels 24:6624-6632. doi:10.1021/ef101223a

Boateng, A.A., D.E. Daugaard, N.M. Goldberg, and K.B. Hicks. 2007. Bench-scale fluidized-bed pyrolysis of switchgrass for bio-oil production. Ind. Eng. Chem. Res. 46:1891-1897. doi:10.1021/ie0614529

Boateng, A.A., C.A. Mullen, N.M. Goldberg, K.B. Hicks, T.E. Devine, I.M. Lima, and J.E. McMurtrey. 2010b. Sustainable production of bioenergy and biochar from the straw of high-biomass soybean lines via fast pyrolysis. Environ. Prog. Sustainable Energy 29:175-183. doi:10.1002/ ep. 10446

Bohn, H.L., B.L. McNeal, and G.A. O’Conner. 1985. Soil chemistry. John Wiley \& Sons, New York.

Bourgois, J., and R. Guyonnet. 1988. Characterization and analysis of torrefied wood. Wood Sci. Technol. 22:143-155. doi:10.1007/BF00355850

Bovey, R.W., and F.R. Miller. 1969. Effect of activated carbon on the phytotoxicity of herbicides in a tropical soil. Weed Sci. 17:189-192.

Brewer, C.E., K. Schmidt-Rohr, J.A. Satrio, and R.C. Brown. 2009. Characterization of biochar from fast pyrolysis and gasification systems. Environ. Prog. Sustainable Energy 28:386-396. doi:10.1002/ep.10378

Bridgeman, T.G., J.M. Jones, I. Shield, and P.T. Williams. 2008. Torrefaction of reed canary grass, wheat straw and willow to enhance solid fuel qualities and combustion properties. Fuel 87:844-856. doi:10.1016/j. fuel.2007.05.041

Bridgeman, T.G., J.M. Jones, A. Williams, and D.J. Waldron. 2010. An investigation of the grindability of two torrefied energy crops. Fuel 89:39113918. doi:10.1016/j.fuel.2010.06.043

Bridgwater, A.V. 1994. Catalysis in thermal biomass conversion. Appl. Catal. A Gen. 116:5-47. doi:10.1016/0926-860X(94)80278-5

Bridgwater, A.V., D. Meier, and D. Radlein. 1999. An overview of fast pyrolysis of biomass. Org. Geochem. 30:1479-1493. doi:10.1016/ S0146-6380(99)00120-5

Brockhoff, S.R., N.E. Christians, R.J. Killorn, R. Horton, and D.D. Davis. 2010. Physical and mineral-nutrition properties of sand-based turfgrass root zones amended with biochar. Agron. J. 102:1627-1631. doi:10.2134/agronj2010.0188

Bruun, E.W., H. Hauggaard-Nielsen, N. Ibrahim, H. Egsgaard, P. Ambus, P.A. Jensen, and K. Dam-Johansen. 2011. Influence of fast pyrolysis temperature on biochar labile fraction and short-term carbon loss in a loamy soil. Biomass Bioenergy 35:1182-1189. doi:10.1016/j. biombioe.2010.12.008

Buol, S.W., R.J. Southard, R.C. Graham, and P.A. McDaniel. 2003. Soil genesis and classification. 5th ed., Iowa State Press, Ames, IA.

Bustin, R.M., and Y. Guo. 1999. Abrupt changes (jumps) in reflectance values and chemical compositions of artificial charcoals and inertinite in coals. Int. J. Coal Geol. 38:237-260. doi:10.1016/S0166-5162(98)00025-1

Cantrell, K.B., and J.H. Martin, II. 2011. State-space temperature regulation of biochar production part II: Application to manure processing via pyrolysis. J. Sci. Food Agric. (in press).

Cao, X.D., and W. Harris. 2010. Properties of dairy-manure-derived biochar pertinent to its potential use in remediation. Bioresour. Technol. 101:5222-5228. doi:10.1016/j.biortech.2010.02.052

Chan, K.Y., L. Van Zwieten, I. Meszaros, A. Downie, and S. Joseph. 2007. Agronomic values of greenwaste biochar as a soil amendment. Aust. J. Soil Res. 45:629-634. doi:10.1071/SR07109

Chang, S.G., and T. Novakov. 1975. Formation of pollution particulate nitrogen compounds by $\mathrm{NO}$-soot and $\mathrm{NH}_{3}$-soot gas-particle surface reactions. Atmos. Environ. 9:495-504. doi:10.1016/0004-6981(75)90109-2

Chen, Y., Y. Shinogi, and M. Taira. 2010. Influence of biochar use on sugarcane growth, soil parameters, and groundwater quality. Aust. J. Soil Res. 48:526-530. doi:10.1071/SR10011

Cheng, C.H., J. Lehmann, and M.H. Engelhard. 2008. Natural oxidation of black carbon in soils: Changes in molecular form and surface charge along a climosequence. Geochim. Cosmochim. Acta 72:1598-1610. doi:10.1016/j.gca.2008.01.010

Cheng, C.H., J. Lehmann, J.E. Thies, S.D. Burton, and M.H. Engelhard. 2006. Oxidation of black carbon by biotic and abiotic processes. Org. Geochem. 37:1477-1488. doi:10.1016/j.orggeochem.2006.06.022

Chidumayo, E.N. 1988. A re-assessment of effects of fire on miombo regeneration in the Zambian Copperbelt. J. Trop. Ecol. 4:361-372. doi:10.1017/ S0266467400003011

Colauto, N.B., A.R.d. Silveira, A.F.d. Eira, and G.A. Linde. 2010. Alternative to peat for Agaricus brasiliensis yield. Bioresour. Technol. 101:712-716. doi:10.1016/j.biortech.2009.08.052

Constantin, M.J., R.R. Henke, and M.A. Mansur. 1977. Effect of activated charcoal on callus growth and shoot organogenesis in tobacco. In Vitro 13:293-296. doi:10.1007/BF02616173

de Beer, J., E. Worrell, and K. Blok. 1998. Future technologies for energy-efficient iron and steel making. Annu. Rev. Energy Environ. 23:123-205. doi:10.1146/annurev.energy.23.1.123

de Keijzer, S., and R.K. Hermann. 1966. Effect of charcoal on germination of Douglas-fir. Northwest Sci. 40:155-163.

Deenik, J.L., T. McClellan, G. Uehara, M.J. Antal, and S. Campbell. 2010. Charcoal volatile matter content influences plant growth and soil nitrogen transformations. Soil Sci. Soc. Am. J. 74:1259-1270. doi:10.2136/ sssaj2009.0115

DeGroot, W.F., T.H. Osterheld, and G.N. Richards. 1991. Chemisorption of oxygen and of nitric oxide on cellulosic chars. Carbon 29:185-195. doi:10.1016/0008-6223(91)90069-U

Devonald, V. 1982. The effect of wood charcoal on the growth and nodulation of garden peas in pot culture. Plant Soil 66:125-127. doi:10.1007/ BF02203411

Ding, Y., Y.-X. Liu, W.-X. Wu, D.-Z. Shi, M. Yang, and Z.-K. Zhong. 2010. Evaluation of biochar effects on nitrogen retention and leaching in multilayered soil columns. Water Air Soil Pollut. 213:47-55. doi:10.1007/ s11270-010-0366-4

Drummond, A.-R.F., and I.W. Drummond. 1996. Pyrolysis of sugar cane bagasse in a wire-mesh reactor. Ind. Eng. Chem. Res. 35:1263-1268. doi:10.1021/ie9503914

Edenborn, H., and D. Severson. 2007. Characterization of waste tar associated with abandoned wood chemical plant sites in Northwest Pennsylvania, USA. Water Air Soil Pollut. 183:331-340. doi:10.1007/ s1 1270-007-9382-4

Egli, D.B., and W. Bruening. 1992. Planting date and soybean yield: Evaluation of environmental effects with a crop simulation model: SOYGRO Agric. For. Meteorol. 62:19-29. doi:10.1016/0168-1923(92)90003-M

Erstfeld, K.M., and J. Snow-Ashbrook. 1999. Effects of chronic low-level $\mathrm{PAH}$ contamination on soil invertebrate communities. Chemosphere 39:2117-2139. doi:10.1016/S0045-6535(98)00421-4

Fernández-Pereira, C., J.A. de la Casa, A. Gómez-Barea, F. Arroyo, C. Leiva, and Y. Luna. 2011. Application of biomass gasification fly ash for brick manufacturing. Fuel 90:220-232. doi:10.1016/j.fuel.2010.07.057

Fink, D.S. 1934. Soil factors which prevent toxicity of calcium cyanamide. J. Am. Soc. Agron. 26:929-939. doi:10.2134/agronj1934.00021962002 600110006x 
Fuertes, A.B., M.C. Arbestain, M. Sevilla, J.A. Maciá-Agulló, S. Fiol, R. López, R.J. Smernik, W.P. Aitkenhead, F. Arce, and F. Macias. 2010. Chemical and structural properties of carbonaceous products obtained by pyrolysis and hydrothermal carbonisation of corn stover. Soil Res. 48:618-626. doi:10.1071/SR10010

Galinato, S.P., J.K. Yoder, and D. Granatstein. 2010. The economic value of biochar in crop production and carbon sequestration. School of Economic Sciences, Washington State University, Pullman, WA. Available at http://www.ses.wsu.edu/PDFFiles/WorkingPapers/sgalinato/WP_201003.pdf (verified 5 Oct. 2011)

Garcia-Montiel, D.C., C. Neill, J. Melillo, S. Thomas, P.A. Steudler, and C.C. Cerri. 2000. Soil phosphorus transformations following forest clearing for pasture in the Brazilian Amazon. Soil Sci. Soc. Am. J. 64:1792-1804. doi:10.2136/sssaj2000.6451792x

Gaskin, J.W., R.A. Speir, K. Harris, K.C. Das, R.D. Lee, L.A. Morris, and D.S. Fisher. 2010. Effect of peanut hull and pine chip biochar on soil nutrients, corn nutrient status, and yield. Agron. J. 102:623-633. doi:10.2134/agronj2009.0083

Gaunt, J.L., and J. Lehmann. 2008. Energy balance and emissions associated with biochar sequestration and pyrolysis bioenergy production. Environ. Sci. Technol. 42:4152-4158. doi:10.1021/es071361i

German, L.A. 2003. Historical contingencies in the coevolution of environment and livelihood: Contributions to the debate on Amazonian Black Earth. Geoderma 111:307-331. doi:10.1016/S0016-7061(02)00270-7

Glaser, B., J. Lehmann, and W. Zech. 2002. Ameliorating physical and chemical properties of highly weathered soils in the tropics with charcoal: A review. Biol. Fertil. Soils 35:219-230. doi:10.1007/s00374-002-0466-4

Goldberg, E.D. 1985. Black carbon in the environment: Properties and distribution. John Wiley \& Sons, New York.

Graber, E., Y. Meller Harel, M. Kolton, E. Cytryn, A. Silber, D. Rav David, L. Tsechansky, M. Borenshtein, and Y. Elad. 2010. Biochar impact on development and productivity of pepper and tomato grown in fertigated soilless media. Plant Soil 337:481-496. doi:10.1007/s11104-010-0544-6

Granatstein, D., C.E. Kruger, H. Collins, S. Galinato, M. Garcia-Perez, and J. Yoder. 2009. Use of biochar from the pyrolysis of waste organic materia as a soil amendment. Final project report, Center for Sustaining Agriculture and Natural Resources, Wenatchee, WA.

Grossman, J.M., B.E. O’Neill, S.M. Tsai, B.Q. Liang, E. Neves, J. Lehmann, and J.E. Thies. 2010. Amazonian Anthrosols support similar microbial communities that differ distinctly from those extant in adjacent, unmodified soils of the same mineralogy. Microb. Ecol. 60:192-205. doi:10.1007/s00248-010-9689-3

Gundale, M., and T. DeLuca. 2007. Charcoal effects on soil solution chemistry and growth of Koeleria macrantha in the ponderosa pine/Douglas-fir ecosystem. Biol. Fertil. Soils 43:303-311. doi:10.1007/s00374-006-0106-5

Haefele, S.M., Y. Konboon, W. Wongboon, S. Amarante, A.A. Maarifat, E.M. Pfeiffer, and C. Knoblauch. 2011. Effects and fate of biochar from rice residues in rice-based systems. Field Crops Res. 121:430-440. doi:10.1016/j.fcr.2011.01.014

Hall, A.D. 1910. The fertility of the soil. Science 32:363-371. doi:10.1126/ science.32.820.363

Hawley, L.F. 1926. Fifty years of wood distillations. Ind. Eng. Chem. 18:929930. doi:10.1021/ie50201a018

Hedges, J.I., G. Eglinton, P.G. Hatcher, D.L. Kirchman, C. Arnosti, S. Derenne, R.P. Evershed, I. Kögel-Knabner, J.W. de Leeuw, R. Littke, W Michaelis, and J. Rullkötter. 2000. The molecularly-uncharacterized component of nonliving organic matter in natural environments. Org. Geochem. 31:945-958. doi:10.1016/S0146-6380(00)00096-6

Herr, D.G., L.C. Duchesne, and R.J. Reader. 1999. Effects of soil organic matter, moisture, shading and ash on white pine (Pinus strobus L.) seedling emergence. New For. 18:219-230. doi:10.1023/A:1006547507369

Hiradate, S., J.F. Ma, and H. Matsumoto. 2007. Strategies of plants to adapt to mineral stresses in problem soils. p. 65-132. In L. Donald Sparks (ed.) Advances in agronomy. Academic Press, Oxford, UK.

Hossain, M.K., V. Strezov, K.Y. Chan, and P.F. Nelson. 2010. Agronomic properties of wastewater sludge biochar and bioavailability of metals in production of cherry tomato (Lycopersicon esculentum). Chemosphere 78:1167-1171. doi:10.1016/j.chemosphere.2010.01.009

Hossain, M.K., V. Strezov, K.Y. Chan, A. Ziolkowski, and P.F. Nelson. 2011. Influence of pyrolysis temperature on production and nutrient properties of wastewater sludge biochar. J. Environ. Manage. 92:223-228. doi:10.1016/j.jenvman.2010.09.008

Huang, Y.F., W.H. Kuan, S.L. Lo, and C.F. Lin. 2008. Total recovery of resources and energy from rice straw using microwave-induced pyrolysis. Bioresour. Technol. 99:8252-8258. doi:10.1016/j.biortech.2008.03.026 Ingham, R.E., J.A. Trofymow, E.R. Ingham, and D.C. Coleman. 1985.
Interactions of bacteria, fungi, and their nematode grazers: Effects on nutrient cycling and plant growth. Ecol. Monogr. 55:119-140. doi: $10.2307 / 1942528$

Insam, H., and M. Seewald. 2010. Volatile organic compounds (VOCs) in soils. Biol. Fertil. Soils 46:199-213. doi:10.1007/s00374-010-0442-3

Inyang, M., B. Gao, P. Pullammanappallil, W.C. Ding, and A.R. Zimmerman. 2010. Biochar from anaerobically digested sugarcane bagasse. Bioresour. Technol. 101:8868-8872. doi:10.1016/j.biortech.2010.06.088

Ippolito, J.A., K.A. Barbarick, and H.A. Elliott. 2011. Drinking water treatment residuals: A review of recent uses. J. Environ. Qual. 40:1-12. doi: $10.2134 /$ jeq2010.0242

Ippolito, J.A., J.M. Novak, D.G. Strawn, K.G. Scheckel, M. Ahmedna, and M.A.S. Niandou. 2012. Macroscopic and molecular investigations of copper sorption by a steam-activated biochar. J. Environ. Qual. doi: $10.2134 /$ jeq 2011.0113

Iswaran, V., K.S. Jauhri, and A. Sen. 1980. Effect of charcoal, coal and peat on the yield of moong, soybean and pea. Soil Biol. Biochem. 12:191-192. doi:10.1016/0038-0717(80)90057-7

Itay, M., C.R. Hill, and D. Glasser. 1989. A study of the low temperature oxidation of coal. Fuel Process. Technol. 21:81-97. doi:10.1016/0378-3820(89)90063-5

Jenny, H. 1946. Arrangement of soil series and types according to functions of soil-forming factors. Soil Sci. 61:375-392. doi:10.1097/00010694-194605000-00005

Jones, D.L., D.V. Murphy, M. Khalid, W. Ahmad, G. Edwards-Jones, and T.H. DeLuca. 2011. Short-term biochar-induced increase in soil CO release is both biotically and abiotically mediated. Soil Biol. Biochem. 43:1723-1731. doi:10.1016/j.soilbio.2011.04

Jones, T.P., W.G. Chaloner, and T.A.G. Kuhlbusch. 1997. Proposed biogeological and chemical based terminology for fire-altered plant matter. p. 9-22. In J.S. Clark, H. Cachier, J.G. Goldhammer, and B. Stocks (ed.) Sediment records of biomass burning and global change: NATO ASI Series, Volume I 51. Springer, New York.

Kadota, M., and Y. Niimi. 2004. Effects of charcoal with pyroligneous acid and barnyard manure on bedding plants. Sci. Hortic. (Amsterdam) 101:327-332. doi:10.1016/j.scienta.2004.01.002

Karaosmanoglu, F., A. Isigigur-Ergundenler, and A. Sever. 2000. Biochar from the straw-stalk of rapeseed plant. Energy Fuels 14:336-339. doi:10.1021/ef9901138

Karagöz, S., T. Bhaskar, A. Muto, Y. Sakata, T. Oshiki, and T. Kishimoto. 2005. Low-temperature catalytic hydrothermal treatment of wood biomass: Analysis of liquid products. Chem. Eng. J. 108:127-137. doi:10.1016/j. cej.2005.01.007

Keiluweit, M., P.S. Nico, M.G. Johnson, and M. Kleber. 2010. Dynamic molecular structure of plant biomass-derived black carbon (biochar). Environ. Sci. Technol. 44:1247-1253. doi:10.1021/es9031419

Khan, M., K.-W. Kim, W. Mingzhi, B.-K. Lim, W.-H. Lee, and J.-Y. Lee. 2008. Nutrient-impregnated charcoal: An environmentally friendly slow-release fertilizer. Environmentalist 28:231-235. doi:10.1007/ s10669-007-9133-5

Khodadad, C.L.M., A.R. Zimmerman, S.J. Green, S. Uthandi, and J.S. Foster. 2011. Taxa-specific changes in soil microbial community composition induced by pyrogenic carbon amendments. Soil Biol. Biochem. 43:385392. doi:10.1016/j.soilbio.2010.11.005

Kim, S.H., S.M. Lee, Y.J. Lee, H.M. Kim, S.Y. Son, and B.H. Song. 2003. Effects of charcoal powder on the growth and development of red pepper and the changes of soil microhlora. Korean J. Organic Agric. 11:55-64.

Kimetu, J.M., and J. Lehmann. 2010. Stability and stabilisation of biochar and green manure in soil with different organic carbon contents. Aust. J. Soil Res. 48:577-585. doi:10.1071/SR10036

Kimetu, J.M., J. Lehmann, S.O. Ngoze, D.N. Mugendi, J.M. Kinyangi, S. Riha, L. Verchot, J.W. Recha, and A.N. Pell. 2008. Reversibility of soil productivity decline with organic matter of differing quality along a degradation gradient. Ecosystems 11:726-739. doi:10.1007/ s10021-008-9154-z

Klasson, K.T., I.M. Lima, L.L. Boihem, and L.H. Wartelle. 2010. Feasibility of mercury removal from simulated flue gas by activated chars made from poultry manures. J. Environ. Manage. 91:2466-2470. doi:10.1016/j. jenvman.2010.06.028

Kratky, B.A., and G.F. Warren. 1971. Activated carbon-vermiculite mixture for increasing herbicide selectivity. Weed Sci. 19:79-81.

Kuhlbusch, T.A.J., and P.J. Crutzen. 1995. Toward a global estimate of black carbon in residues of vegetation fires representing a sink of atmospheric $\mathrm{CO}_{2}$ and a source of $\mathrm{O}_{2}$. Global Biogeochem. Cycles 9:491-501. doi:10.1029/95GB02742

Kulmatiski, A., and K.H. Beard. 2006. Activated carbon as a restoration tool: 
Potential for control of invasive plants in abandoned agricultural fields. Restor. Ecol. 14:251-257. doi:10.1111/j.1526-100X.2006.00127.x

Laird, D., P. Fleming, B.Q. Wang, R. Horton, and D. Karlen. 2010a. Biochar impact on nutrient leaching from a Midwestern agricultural soil. Geoderma 158:436-442. doi:10.1016/j.geoderma.2010.05.012

Laird, D.A. 2008. The charcoal vision: A win-win-win scenario for simultaneously producing bioenergy, permanently sequestering carbon, while improving soil and water quality. Agron. J. 100:178-181. doi:10.2134/ agrojn12007.0161

Laird, D.A., P. Fleming, D.D. Davis, R. Horton, B.Q. Wang, and D.L. Karlen. 2010b. Impact of biochar amendments on the quality of a typical Midwestern agricultural soil. Geoderma 158:443-449. doi:10.1016/j. geoderma.2010.05.013

Lau, J.A., K.P. Puliafico, J.A. Kopshever, H. Steltzer, E.P. Jarvis, M. Schwarzländer, S.Y. Strauss, and R.A. Hufbauer. 2008. Inference of allelopathy is complicated by effects of activated carbon on plant growth. New Phytol. 178:412-423. doi:10.1111/j.1469-8137.2007.02360.x

Lee, J.W., M. Kidder, B.R. Evans, S. Paik, A.C. Buchanan, III, C.T. Garten, and R.C. Brown. 2010. Characterization of biochars produced from cornstovers for soil amendment. Environ. Sci. Technol. 44:7970-7974. doi:10.1021/es101337x

Lefroy, J.H. 1883. Remarks on the chemical analyses of samples of soil from Bermuda. Foreign and Commonwealth Office Collection, Royal Gazette, Hamilton, ON, Canada.

Lehmann, J. 2007. Bio-energy in the black. Front. Ecol. Environ 5:381-387. doi:10.1890/1540-9295(2007)5[381:BITB]2.0.CO;2

Lehmann, J., and S. Joseph. 2009. Biochar for environmental management science and technology. Earthscan, London.

Lehmann, J., M. Rillig, J. Thies, C.A. Masiello, W.C. Hockaday, and D. Crowley. 2011. Biochar effects on soil biota: A review. Soil Biol. Biochem. 43:1812-1836

Lei, H.W., S.J. Ren, and J. Julson. 2009. The effects of reaction temperature and time and particle size of corn stover on microwave pyrolysis. Energy Fuels 23:3254-3261. doi:10.1021/ef9000264

Leibundgut, H. 1960. The effect of charcoal on the germination and development of spruce, pine and larch. Schweizerische Zeitschrift fur Forstwesen 111:172-178.

Liang, C.S., Z. Dang, B.H. Mao, W.L. Huang, and C.Q. Liu. 2006. Equilibrium sorption of phenanthrene by soil humic acids. Chemosphere 63:1961-1968. doi:10.1016/j.chemosphere.2005.09.065

Lima, I.M., A.A. Boateng, and K.T. Klasson. 2010. Physicochemical and adsorptive properties of fast-pyrolysis bio-chars and their steam activated counterparts. J. Chem. Technol. Biotechnol. 85:1515-1521.

Lima, I.M., and W.E. Marshall. 2009. Activated carbons from animal manure. U.S. Patent 7524,795. Date issued: 28 Apr. 2009.

Lima, I.M., and W.E. Marshall. 2010. Pyrolytic products from poultry manure. U.S. Patent 7794,601. Date issued: 14 Sept. 2010.

Linscott, D.L., and R.D. Hagin. 1967. Protecting alfalfa seedlings from a triazine with activated charcoal. Weeds 15:304-306. doi:10.2307/4040994

Lussier, M.G., J.C. Shull, and D.J. Miller. 1994. Activated carbon from cherry stones. Carbon 32:1493-1498. doi:10.1016/0008-6223(94)90144-9

Major, J., J. Lehmann, M. Rondon, and C. Goodale. 2010a. Fate of soil-applied black carbon: Downward migration, leaching and soil respiration. Glob. Change Biol. 16:1366-1379. doi:10.1111/j.1365-2486.2009.02044.x

Major, J., M. Rondon, D. Molina, S.J. Riha, and J. Lehmann. $2010 \mathrm{~b}$. Maize yield and nutrition during 4 years after biochar application to a Colombian savanna oxisol. Plant Soil 333:117-128. doi:10.1007/ s11104-010-0327-0

Makoto, K., D. Choi, Y. Hashidoko, and T. Koike. 2011. The growth of Larix gmelinii seedlings as affected by charcoal produced at two different temperatures. Biol. Fertil. Soils 47:467-472.

Mann, C.C. 2005. 1491: New revelations of the Americas before Columbus. Vintage and Anchor Books, New York.

Masclet, P., M.A. Bresson, and G. Mouvier. 1987. Polycyclic aromatic hydrocarbons emitted by power stations, and influence of combustion conditions. Fuel 66:556-562. doi:10.1016/0016-2361(87)90163-3

Masiello, C.A. 2004. New directions in black carbon organic geochemistry. Mar. Chem. 92:201-213. doi:10.1016/j.marchem.2004.06.043

Masiello, C.A., E.R.M. Druffel, and L.A. Currie. 2002. Radiocarbon measurements of black carbon in aerosols and ocean sediments. Geochim. Cosmochim. Acta 66:1025-1036. doi:10.1016/S0016-7037(01)00831-6

Mason, J.R., and L. Clark. 1994. Use of activated charcoal and other particulate substances as feed additives to suppress bird feeding. Crop Prot. 13:219-224. doi:10.1016/0261-2194(94)90081-7

Mason, J.R., and L. Clark. 1995. Evaluation of methyl anthranilate and acti- vated charcoal as snow goose grazing deterrents. Crop Prot. 14:467-469. doi:10.1016/0261-2194(95)00027-J

McCarl, B.A., C. Peacocke, R. Chrisman, C. Kung, and R.D. Sands. 2009. Economics of biochar production, utilization and greenhouse gas offsets. p. 341-358. In J. Lehmann and S. Joseph (ed.) Biochar for environmental management. Earthscan, London.

McLauchlan, K. 2006. The nature and longevity of agricultural impacts on soil carbon and nutrients: A review. Ecosystems 9:1364-1382. doi:10.1007/ s10021-005-0135-1

Menéndez, J.A., A. Domínguez, Y. Fernández, and J.J. Pis. 2006. Evidence of self-gasification during the microwave-induced pyrolysis of coffee hulls. Energy Fuels 21:373-378. doi:10.1021/ef060331i

Mercedes Maroto-Valer, M., Z. Lu, Y. Zhang, and Z. Tang. 2008. Sorbents for $\mathrm{CO}_{2}$ capture from high carbon fly ashes. Waste Manag. 28:2320-2328. doi:10.1016/j.wasman.2007.10.012

Milosavljevic, I., V. Oja, and E.M. Suuberg. 1996. Thermal effects in cellulose pyrolysis: Relationship to char formation processes. Ind. Eng. Chem. Res. 35:653-662. doi:10.1021/ie9504381

Mishra, B.K., and P.S. Ramakrishnan. 1983. Slash and burn agriculture at higher elevations in North-Eastern India. I. Sediment, water and nutrient losses. Agric. Ecosyst. Environ. 9:69-82. doi:10.1016/0167-8809(83)90007-5

Mohamed-Yasseen, Y. 2001. Influence of agar and activated charcoal on uptake of gibberellin and plant morphogenesis in vitro. Cellular Develop. Bio. Plant 37:204-205.

Mok, W.S.L., and M.J. Antal, Jr. 1983. Effects of pressure on biomass pyrolysis. II. Heats of reaction of cellulose pyrolysis. Thermochim. Acta 68:165-186. doi:10.1016/0040-6031(83)80222-6

Møller, A.P., and M.D. Jennions. 2001. Testing and adjusting for publication bias. Trends Ecol. Evol. 16:580-586. doi:10.1016/ S0169-5347(01)02235-2

Molton, P.M., K.R. Miller, A.J. Russell, and M.J. Donovan. 1981. Effects of reaction conditions on the aqueous thermochemical conversion of biomass to oil. p. 137-162. In Biomass as a nonfossil fuel source. American Chemical Society, Washington, DC.

Mullen, C.A., A.A. Boateng, K.B. Hicks, N.M. Goldberg, and R.A. Moreau. 2010. Analysis and comparison of bio-oil produced by fast pyrolysis from three barley biomass/byproduct streams. Energy Fuels 24:699-706. doi:10.1021/ef900912s

Namgay, T., B. Singh, and B.P. Singh. 2010. Influence of biochar application to soil on the availability of $\mathrm{As}, \mathrm{Cd}, \mathrm{Cu}, \mathrm{Pb}$, and $\mathrm{Zn}$ to maize (Zea mays L.). Aust. J. Soil Res. 48:638-647. doi:10.1071/SR10049

Ng, C., J.N. Losso, W.E. Marshall, and R.M. Rao. 2002. Freundlich adsorption isotherms of agricultural by-product-based powdered activated carbons in a geosmin-water system. Bioresour. Technol. 85:131-135. doi:10.1016/S0960-8524(02)00093-7

Ng, C., W.E. Marshall, R.M. Rao, R.R. Bansode, and J.N. Losso. 2003. Activated carbon from pecan shell: Process description and economic analysis. Ind. Crops Prod. 17:209-217. doi:10.1016/S0926-6690(03)00002-5

Nguyen, B.T., J. Lehmann, W.C. Hockaday, S. Joseph, and C.A. Masiello. 2010. Temperature sensitivity of black carbon decomposition and oxidation. Environ. Sci. Technol. 44:3324-3331. doi:10.1021/es903016y

Noguera, D., M. Rondon, K.R. Laossi, V. Hoyos, P. Lavelle, M.H.C. de Carvalho, and S. Barot. 2010. Contrasted effect of biochar and earthworms on rice growth and resource allocation in different soils. Soil Biol. Biochem. 42:1017-1027. doi:10.1016/j.soilbio.2010.03.001

Novak, J.M., and W.J. Busscher. 2011. Selection and use of designer biochars to improve characteristics of Southeastern USA Coastal Plain soils. In J.E. Lee (ed.) Advanced biofuels and bioproducts. Springer Science, New York.

Novak, J.M., W.J. Busscher, D.L. Laird, M. Ahmedna, D.W. Watts, and M.A.S. Niandou. 2009a. Impact of biochar amendment on fertility of a Southeastern coastal plain soil. Soil Sci. 174:105-112. doi:10.1097/ SS.0b013e3181981d9a

Novak, J.M., I. Lima, B. Xing, J.W. Gaskin, C. Steiner, K.C. Das, M. Ahmedna, D. Rehrah, D.W. Watts, W.J. Busscher, and H. Schomberg. 2009b. Characterization of designer biochar produced at different temperatures and their effects on a loamy sand. Ann. Environ. Sci. 3:195-206.

Novakov, T., S.G. Chang, and A.B. Harker. 1974. Sulfates as pollution particulates: Catalytic formation on carbon (soot) particles. Science 186:259261. doi: $10.1126 /$ science. 186.4160 .259

Nuithitikul, K., S. Srikhun, and S. Hirunpraditkoon. 2010. Influences of pyrolysis condition and acid treatment on properties of durian peel-based activated carbon. Bioresour. Technol. 101:426-429. doi:10.1016/j. biortech.2009.07.040

Nutman, P.S. 1952. Host factors influencing infection and nodule development in leguminous plants. Proc. Royal Soc. Lon. Ser. B Biol. Science 
139:176-185.

O’Neill, B., J. Grossman, M.T. Tsai, J.E. Gomes, J. Lehmann, J. Peterson, E. Neves, and J.E. Thies. 2009. Bacterial community composition in brazilian anthrosols and adjacent soils characterized using culturing and molecular identification. Microb. Ecol. 58:23-35. doi:10.1007/ s00248-009-9515-y

O'Toole, A. 2010. The agronomic effects of biochar and the implications for improving environmental outcomes in norwegian agriculture. Department of International Environment and Development Studies, Norwegian Univ. of Life Sciences, Oslo, Norway.

Ogawa, M., and Y. Okimori. 2010. Pioneering works in biochar research: Japan. Soil Res. 48:489-500. doi:10.1071/SR10006

Oguntunde, P., M. Fosu, A. Ajayi, and N. Giesen. 2004. Effects of charcoal production on maize yield, chemical properties and texture of soil. Biol. Fertil. Soils 39:295-299. doi:10.1007/s00374-003-0707-1

Owen, E.W. 1975. Trek of the oil finders: A history of exploration for petroleum. American Association of Petroleum Geologists Memoir, Tulsa, $\mathrm{OK}$.

Pérez-Piqueres, A., V. Edel-Hermann, C. Alabouvette, and C. Steinberg. 2006. Response of soil microbial communities to compost amendments. Soil Biol. Biochem. 38:460-470. doi:10.1016/j.soilbio.2005.05.025

Phanphanich, M., and S. Mani. 2011. Impact of torrefaction on the grindability and fuel characteristics of forest biomass. Bioresour. Technol. 102:1246-1253. doi:10.1016/j.biortech.2010.08.028

Prins, M.J., K.J. Ptasinski, and F.J.J.G. Janssen. 2006. Torrefaction of wood: Part 1. Weight loss kinetics. J. Anal. Appl. Pyrolysis 77:28-34. doi:10.1016/j.jaap.2006.01.002

Ptasinski, K.J. 2008. Thermodynamic efficiency of biomass gasification and biofuels conversion. Biofuels Bioprod. Biorefin. 2:239-253. doi:10.1002/bbb.65

Puri, B., D.D. Singh, J. Nath, and L. Sharma. 1958. Chemisorption of oxygen on activated charcoal and sorption of acids and bases. Ind. Eng. Chem. 50:1071-1074. doi:10.1021/ie50583a046

Pütün, A.E., E. Önal, B.B. Uzun, and N. Özbay. 2007. Comparison between the "slow" and "fast" pyrolysis of tobacco residue. Ind. Crops Prod. 26:307-314. doi:10.1016/j.indcrop.2007.03.011

Rajkovich, S. 2010. Biochar as an amendment to improve soil fertility. Research Honors thesis, Cornell University, Ithaca, NY.

Repellin, V., A. Govin, M. Rolland, and R. Guyonnet. 2010. Energy requirement for fine grinding of torrefied wood. Biomass Bioenergy 34:923930. doi:10.1016/j.biombioe.2010.01.039

Richter, D.D., Jr. 2007. Humanity's transformation of earth's soil: Pedology's new frontier. Soil Sci. 172:957-967. doi:10.1097/ss.0b013e3181586bb7

Rillig, M.C., M. Wagner, M. Salem, P.M. Antunes, C. George, H.G. Ramke, M.M. Titirici, and M. Antonietti. 2010. Material derived from hydrothermal carbonization: Effects on plant growth and arbuscular mycorrhiza. Appl. Soil Ecol. 45:238-242. doi:10.1016/j.apsoil.2010.04.011

Roberts, K.G., B.A. Gloy, S. Joseph, N.R. Scott, and J. Lehmann. 2010. Life cycle assessment of biochar systems: Estimating the energetic, economic, and climate change potential. Environ. Sci. Technol. 44:827-833. doi:10.1021/es902266r

Rondon, M.A., J. Lehmann, J. Ramirez, and M. Hurtado. 2007. Biological nitrogen fixation by common beans (Phaseolus vulgaris L.) increases with bio-char additions. Biol. Fertil. Soils 43:699-708. doi:10.1007/ s00374-006-0152-z

Rosenfeld, P.E. 2001. Effect of high carbon ash on biosolids odor emissions and microbial activity. Water Air Soil Pollut. 131:245-260. doi:10.1023/A:1011975732056

Rosenfeld, P.E., and C.L. Henry. 2001. Activated carbon and wood ash sorption of wastewater, compost, and biosolids odorants. Water Environ. Res. 73:388-393. doi:10.2175/106143001X139425

Rutto, K.L., and F. Mizutani. 2006. Effect of mycorrhizal inoculation and activated charcoal on growth and nutrition in peach (Prunus persica Batsch) seedlings treated with peach root-bark extracts. J. Jpn. Soc. Hortic. Sci. 75:463-468. doi:10.2503/jjshs.75.463

Salleh, M.A.M., N.H. Kisiki, H.M. Yusuf, and W. Ghani. 2010. Gasification of biochar from empty fruit bunch in a fluidized bed reactor. Energies 3:1344-1352. doi:10.3390/en3071344

Shneour, E.A. 1966. Oxidation of graphitic carbon in certain soils. Science 151:991-992. doi:10.1126/science.151.3713.991

Singh, B., B.P. Singh, and A.L. Cowie. 2010a. Characterisation and evaluation of biochars for their application as a soil amendment. Aust. J. Soil Res. 48:516-525. doi:10.1071/SR10058

Singh, B.P., B.J. Hatton, B. Singh, A.L. Cowie, and A. Kathuria. 2010b. Influence of biochars on nitrous oxide emission and nitrogen leaching from two contrasting soils. J. Environ. Qual. 39:1224-1235. doi:10.2134/ jeq2009.0138

Sjöström, E. 1993. Wood chemistry: Fundamentals and applications. Academic Press, New York.

Skjemstad, J.O., D.C. Reicosky, A.R. Wilts, and J.A. McGowan. 2002. Charcoal carbon in U.S. agricultural soils. Soil Sci. Soc. Am. J. 66:12491255. doi: $10.2136 /$ sssaj2002.1249

Sohi, S.P., E. Krull, E. Lopez-Capel, and R. Bol. 2010. A review of biochar and its use and function in soil. p. 47-82. In Donald L. Sparks (ed.) Advances in agronomy. Academic Press, Waltham, MA.

Solaiman, Z.M., P. Blackwell, L.K. Abbott, and P. Storer. 2010. Direct and residual effect of biochar application on mycorrhizal root colonisation, growth and nutrition of wheat. Aust. J. Soil Res. 48:546-554. doi: $10.1071 /$ SR 10002

Song, J.Z., and P.A. Peng. 2010. Characterisation of black carbon materials by pyrolysis-gas chromatography-mass spectrometry. J. Anal. Appl. Pyrolysis 87:129-137. doi:10.1016/j.jaap.2009.11.003

Spokas, K.A. 2010. Review of the stability of biochar in soils: Predictability of O:C molar ratios. Carbon Manage. 1:289-303. doi:10.4155/cmt.10.32

Spokas, K., and D. Reicosky. 2009. Impacts of sixteen different biochars on soil greenhouse gas production. Ann. Environ. Sci. 3:179-193.

Spokas, K.A., J.M. Baker, and D.C. Reicosky. 2010. Ethylene: Potential key for biochar amendment impacts. Plant Soil 333:443-452. doi:10.1007/ s11104-010-0359-5

Spokas, K.A., W.C. Koskinen, J.M. Baker, and D.C. Reicosky. 2009. Impacts of woodchip biochar additions on greenhouse gas production and sorption/degradation of two herbicides in a Minnesota soil. Chemosphere 77:574-581. doi:10.1016/j.chemosphere.2009.06.053

Spokas, K.A., J.M. Novak, C.E. Stewart, K.B. Cantrell, M. Uchimiya, M.G. duSaire, and K.S. Ro. 2011. Qualitative analysis of volatile organic compounds on biochar. Chemosphere 85:869-882. doi:10.1016/j. chemosphere

Steinbeiss, S., G. Gleixner, and M. Antonietti. 2009. Effect of biochar amendment on soil carbon balance and soil microbial activity. Soil Biol. Biochem. 41:1301-1310. doi:10.1016/j.soilbio.2009.03.016

Steiner, C., K.C. Das, N. Melear, and D. Lakly. 2010. Reducing nitrogen loss during poultry litter composting using biochar. J. Environ. Qual. 39:1236-1242. doi: $10.2134 /$ jeq2009.0337

Steiner, C., W.G. Teixeira, J. Lehmann, T. Nehls, J.L.V. de Macedo, W.E.H. Blum, and W. Zech. 2007. Long term effects of manure, charcoal and mineral fertilization on crop production and fertility on a highly weathered Central Amazonian upland soil. Plant Soil 291:275-290. doi:10.1007/s11104-007-9193-9

Streubel, J., H. Collins, D. Granastein, and C. Kruger. 2010. Biochar sorption of phosphorus from dairy lagoons. Presented at the ASA, CSSA, and SSSA 2010 International Annual Meetings, 31 Oct.-4 Nov. 2010, Long Beach, CA. Abstract accessable at http://a-c-s.confex.com/ crops/2010am/webprogram/Paper58223.html (verified 5 Oct. 2011).

Suhardi, E., E. Faridah, E. Iskandar, and S. Rahayu. 2006. Mycorrhizal formation and growth of Shorea leprosula in Bukit Suharto after using charcoal and rockphosphate. p. 175-183. In Suzuki K., et al. (ed.) Plantation technology in tropical forest science. Springer, Tokyo.

Sullivan, T.S., M.E. Stromberger, and M.W. Paschke. 2006. Parallel shifts in plant and soil microbial communities in response to biosolids in a semi-arid grassland. Soil Biol. Biochem. 38:449-459. doi:10.1016/j. soilbio.2005.05.018

Sumner, M.E. 2000. Beneficial use of effluents, wastes, and biosolids. Commun. Soil Sci. Plant Anal. 31:1701-1715. doi:10.1080/00103620009370532

Tagoe, S.O., T. Horiuchi, and T. Matsui. 2008. Preliminary evaluation of the effects of carbonized chicken manure, refuse derived fuel and $\mathrm{K}$ fertilizer application on the growth, nodulation, yield, $\mathrm{N}$ and $\mathrm{P}$ contents of soybean and cowpea in the greenhouse. African J. Agric. Res. 3:759-774.

Tavares, P., and P. Barbeira. 2008. Influence of pencil lead hardness on voltammetric response of graphite reinforcement carbon electrodes. J. Appl. Electrochem. 38:827-832. doi:10.1007/s10800-008-9518-2

Tejerina, M.R. 2010. Biochar as a strategy for sustainable land management, poverty reduction and climate change mitigation/adaptation: Thermolysis of lignin for value-added products. Institute of Environmental Studies (Instituut vor Milieustudies-IVM), Vrije Universiteit, Amsterdam, the Netherlands.

Topoliantz, S., J.-F. Ponge, and S. Ballof. 2005. Manioc peel and charcoal: A potential organic amendment for sustainable soil fertility in the tropics. Biol. Fertil. Soils 41:15-21. doi:10.1007/s00374-004-0804-9

Tryon, E.H. 1948. Effect of charcoal on certain physical, chemical, and biological properties of forest soils. Ecol. Monogr. 18:81-115. doi: $10.2307 / 1948629$ 
Turner, E.R. 1955. The effect of certain adsorbents on the nodulation of clover plants. Ann. Bot. (Lond.) 19:149-160.

Uchimiya, M., K.T. Klasson, L.H. Wartelle, and I.M. Lima. 2011. Influence of soil properties on heavy metal sequestration by biochar amendment: 1. Copper sorption isotherms and the release of cations. Chemosphere 82:1431-1437. doi:10.1016/j.chemosphere.2010.11.050

Vaccari, F.P., S. Baronti, E. Lugato, L. Genesio, S. Castaldi, F. Fornasier, and F. Miglietta. 2011. Biochar as a strategy to sequester carbon and increase yield in durum wheat. Eur. J. Agron. 34:231-238. doi:10.1016/j. eja.2011.01.006

van Duck, P.J., and H. van de Voorde. 1984. Activated charcoal and microflora in water treatment. Water Res. 18:1361-1364. doi:10.1016/0043-1354(84)90004-6

van Zwieten, L., S. Kimber, S. Morris, A. Downie, E. Berger, J. Rust, and C. Scheer. 2010a. Influence of biochars on flux of $\mathrm{N}_{2} \mathrm{O}$ and $\mathrm{CO}_{2}$ from Ferrosol. Aust. J. Soil Res. 48:555-568. doi:10.1071/SR10004

Van Zwieten, L., S. Kimber, S. Morris, K.Y. Chan, A. Downie, J. Rust, S. Joseph, and A. Cowie. 2010b. Effects of biochar from slow pyrolysis of papermill waste on agronomic performance and soil fertility. Plant Soil 327:235-246. doi:10.1007/s11104-009-0050-x

Vantsis, J.T., and G. Bond. 1950. The effect of charcoal on the growth of leguminous plants in sand culture. Ann. Appl. Biol. 37:159-168. doi:10.1111/j.1744-7348.1950.tb01036.x

Verheijen, F.G.A., S. Jeffery, A.C. Bastos, M. van der Velde, and I. Diafas. 2009. Biochar application to soils: A critical scientific review of effects on soil properties, processes and functions. Office for the Official Publications of the European Communities, Luxembourg.

Wallis, C., C.H. Stagg, and J.L. Melnick. 1974. The hazards of incorporating charcoal filters into domestic water systems. Water Res. 8:111-113. doi:10.1016/0043-1354(74)90135-3

Wan, H.B., H. Chi, M.K. Wong, and C.Y. Mok. 1994. Solid-phase microextraction using pencil lead as sorbent for analysis of organic pollutants in water. Anal. Chim. Acta 298:219-223. doi:10.1016/0003-2670(94)00270-3

Wang, J., and A.-N. Kawde. 2001. Pencil-based renewable biosensor for labelfree electrochemical detection of DNA hybridization. Anal. Chim. Acta 431:219-224. doi:10.1016/S0003-2670(00)01318-0

Wang, P.J., and L.C. Huang. 1976. Beneficial effects of activated charcoal on plant tissue and organ cultures. In Vitro 12:260-262. doi:10.1007/ BF02796447

Warnock, D.D., J. Lehmann, T.W. Kuyper, and M.C. Rillig. 2007. Mycorrhizal responses to biochar in soil: Concepts and mechanisms. Plant Soil 300:9-20. doi:10.1007/s11104-007-9391-5

Warnock, D.D., D.L. Mummey, B. McBride, J. Major, J. Lehmann, and M.C. Rillig. 2010. Influences of non-herbaceous biochar on arbuscular mycorrhizal fungal abundances in roots and soils: Results from growth-chamber and field experiments. Appl. Soil Ecol. 46:450-456. doi:10.1016/j. apsoil.2010.09.002

Whitman, T., and C. Lehmann. 2011. Systematic under- and overestimation of GHG reductions in renewable biomass systems. Clim. Change 104:415-422. doi:10.1007/s10584-010-9984-5

Yamato, M., Y. Okimori, I.F. Wibowo, S. Anshori, and M. Ogawa. 2006. Effects of the application of charred bark of Acacia mangium on the yield of maize, cowpea and peanut, and soil chemical properties in South Sumatra, Indonesia. Soil Sci. Plant Nutr. 52:489-495. doi:10.1111/j.1747-0765.2006.00065.x

Yao, F.X., M.C. Arbestain, S. Virgel, F. Blanco, J. Arostegui, J.A. Macia-Agullo, and F. Macias. 2010. Simulated geochemical weathering of a mineral ash-rich biochar in a modified Soxhlet reactor. Chemosphere 80:724732. doi:10.1016/j.chemosphere.2010.05.026

Yip, K., H. Wu, and D.K. Zhang. 2007. Effect of inherent moisture in collie coal during pyrolysis due to in-situ steam gasification. Energy Fuels 21:2883-2891. doi:10.1021/ef7002443

Yip, K., F.J. Tian, J. Hayashi, and H.W. Wu. 2010. Effect of alkali and alkaline earth metallic species on biochar reactivity and syngas compositions during steam gasification. Energy Fuels 24:173-181. doi:10.1021/ ef $900534 n$

Yoder, J., and S. Galinato. 2009. Economic tradeoff between biochar and biooil production via pyrolysis. p. 59-75. In D. Granatstein et al. (ed.) Use of biochar from the pyrolysis of waste organic material as a soil amendment. Final Project Report, Center for Sustaining Agriculture and Natural Resources, Washington State Univ., Wenatchee.

Yuan, X.Z., J.Y. Tong, G.M. Zeng, H. Li, and W. Xie. 2009. Comparative studies of products obtained at different temperatures during straw liquefaction by hot compressed water. Energy Fuels 23:3262-3267. doi:10.1021/ef900027d

Zak, D.R., W.E. Holmes, D.C. White, D.P. Aaron, and D. Tilman. 2003. Plant diversity, soil microbial communities, and ecosystem function: Are there any links? Ecology 84:2042-2050. doi:10.1890/02-0433

Zhang, A., L. Cui, G. Pan, L. Li, Q. Hussain, X. Zhang, J. Zheng, and D. Crowley. 2010. Effect of biochar amendment on yield and methane and nitrous oxide emissions from a rice paddy from Tai Lake plain, China. Agric. Ecosyst. Environ. 139:469-475.

Zhang, Q., J. Chang, T. Wang, and Y. Xu. 2007. Review of biomass pyrolysis oil properties and upgrading research. Energy Convers. Manage. 48:8792. doi:10.1016/j.enconman.2006.05.010

Zimmerman, A.R. 2010. Abiotic and microbial oxidation of laboratory-produced black carbon (biochar). Environ. Sci. Technol. 44:1295-1301. doi:10.1021/es903140c

Zimmerman, A.R., B. Gao, and M.-Y. Ahn. 2011. Positive and negative carbon mineralization priming effects among a variety of biocharamended soils. Soil Biol. Biochem. 43:1169-1179 . doi:10.1016/j. soilbio.2011.02.005 